University of Nebraska - Lincoln

DigitalCommons@University of Nebraska - Lincoln

2012

\title{
LES of temporally evolving mixing layers by an eighth-order filter scheme
}

Abdellah Hadjadj

Fluid Mechanics, hadjadj@coria.fr

Helen C. Yee

Ames Research Center, yee@nas.nasa.gov

Bjorn Sjögreen

Lawrence Livermore National Laboratories, sjogreen2@llnl.gov

Follow this and additional works at: https://digitalcommons.unl.edu/nasapub

Hadjadj, Abdellah; Yee, Helen C.; and Sjögreen, Bjorn, "LES of temporally evolving mixing layers by an eighth-order filter scheme" (2012). NASA Publications. 113.

https://digitalcommons.unl.edu/nasapub/113

This Article is brought to you for free and open access by the National Aeronautics and Space Administration at DigitalCommons@University of Nebraska - Lincoln. It has been accepted for inclusion in NASA Publications by an authorized administrator of DigitalCommons@University of Nebraska - Lincoln. 


\title{
LES of temporally evolving mixing layers by an eighth-order filter scheme
}

\author{
Abdellah Hadjadj ${ }^{1, \ddagger}$, Helen C. Yee ${ }^{2, *, \dagger}$ and Bjorn Sjögreen ${ }^{3}$ \\ ${ }^{1}$ Fluid Mechanics, CORIA UMR 6614 CNRS, INSA de Rouen, 76800 St-Etienne du Rouvray, France \\ ${ }^{2}$ Ames Research Center, NASA, Moffett Field, CA 94035, USA \\ ${ }^{3}$ CASC, Lawrence Livermore National Laboratories, Livermore, CA 94551, USA
}

\begin{abstract}
SUMMARY
An eighth-order filter method for a wide range of compressible flow speeds (H. C. Yee and B. Sjogreen, Proceedings of ICOSAHOM09, June 22-26, 2009, Trondheim, Norway) is employed for large eddy simulations (LES) of temporally evolving mixing layers (TML) for different convective Mach numbers $\left(M_{C}\right)$ and Reynolds numbers. The high-order filter method is designed for accurate and efficient simulations of shock-free compressible turbulence, turbulence with shocklets, and turbulence with strong shocks with minimum tuning of scheme parameters. The value of the $M_{c}$ considered is for the TML range from the quasi-incompressible regime to the highly compressible supersonic regime. The three main characteristics of compressible TML (the self-similarity property, compressibility effects, and the presence of large-scale structures with shocklets for high $M_{c}$ ) are considered for the LES study. The LES results that used the same scheme parameters for all studied cases agree well with experimental results and published direct numerical simulations (DNS). Published 2012. This article is a US Government work and is in the public domain in the USA.
\end{abstract}

Received 22 November 2011; Accepted 28 November 2011

KEY WORDS: $\quad$ DNS; LES; homogeneous turbulence; mixing layer; SWBLI

\section{MOTIVATION AND OBJECTIVE}

In the last decade, there has been an increase in the use of computational fluid dynamics (CFD) in engineering science, not only for fundamental understanding of complex compressible turbulent physics but also for the development and design of industrial devices. Because of the recent progress in petascale computing, in tandem with advances in algorithm development for accurate direct numerical simulations (DNS) and large eddy simulations (LES) of shock-free compressible turbulence and turbulence with strong shocks, this type of DNS and LES computation has gradually been able to tackle more complex flow physics. Advances in flow visualization tools have paved the way to extracting valuable information from the computed results containing hundreds of terabytes of data. Examples include flows through internal propulsive nozzles with shock-wave propagation or sound emission from supersonic jets, and mixing and shock/boundary layer interactions.

In compressible turbulent combustion/nonequilibrium flows, the construction of numerical schemes for (i) stable and accurate simulation of turbulence with strong shocks and the construction of numerical schemes for (ii) the procurement of the correct propagation speed of discontinuities for stiff reacting terms on 'coarse grids' share one important ingredient-minimization of numerical dissipation while maintaining numerical stability. Here, 'coarse grids' means the standard mesh

\footnotetext{
*Correspondence to: Helen C. Yee, Ames Research Center, NASA, Moffett Field, CA 94035, USA.

†E-mail: yee@nas.nasa.gov

† This work was performed when the first author was a visiting scholar at the Center for Turbulence Research, Stanford University.
} 
density requirement for the accurate simulation of typical nonreacting flows. This dual requirement to achieve both numerical stability and accuracy with zero or minimal use of numerical dissipation is most often conflicting with existing schemes that were designed for nonreacting flows. In addition to the minimization of numerical dissipation, while maintaining numerical stability in compressible turbulence with strong shocks, Yee and Sjögreen, Yee, and Yee and Sweby [1-6] discussed a general framework for the design of such schemes. Yee and Sjögreen [7], Sjögreen and Yee [8], Wei et al. [9], and references cited therein present their recent progress on the subject. In [10], a short overview of this recent progress is given. The discussion addresses three separate, yet interwoven, types of numerical challenges for high-speed turbulent reacting flows containing discontinuities. This paper is confined to the study of turbulent mixing for nonreacting flows. The study for turbulent mixing for reacting flows is planned.

\subsection{Recent progress in numerical methods for turbulence with strong shocks}

The current trends in the containment of numerical dissipation in the DNS and LES of turbulence with shocks are summarized in $[6,7,11]$. See the cited references for details on these current trends. Before presenting the temporally evolving mixing layers (TML) studies, the key ingredients and the performance of the high-order nonlinear filter schemes with preprocessing and postprocessing steps, in conjunction with the use of a high-order nondissipative spatial base scheme [7,11], are briefly illustrated for two test cases.

1.1.1. High-order nonlinear filter schemes [5, 7, 11, 12]. Before the application of a high-order nondissipative spatial base scheme, the preprocessing step that improves stability had split the inviscid flux derivatives of the governing equation(s) in three ways, depending on the flow types and the desire for rigorous mathematical analysis or physical argument.

- Entropy splitting of Olsson and Oliger [13] and Yee et al. $[3,14]$ The resulting form is nonconservative, and the derivation is based on the entropy norm stability with numerical boundary closure for the initial value boundary problem.

- The system form of the Ducros et al. splitting [15]. This is a conservative splitting, and the derivation is based on physical arguments.

- Tadmor entropy conservation formulation for systems (Sjögreen \& Yee [16]). The derivation is based on mathematical analysis. It is a generalization of Tadmor's entropy formulation of systems and has not been fully tested on complex flows.

After the application of a nondissipative high-order spatial base scheme on the split form of the governing equation(s), for the improvement of the nonlinear stability of the nondissipative spatial base scheme, the postprocessing step of Yee and Sjögreen [5,7], and Sjögreen and Yee [12] nonlinearly filtered the solution with the use of the dissipative portion of a high-order shock-capturing scheme with a local flow sensor. These flow sensors provide locations and amounts of built-in shockcapturing dissipation that can be further reduced or eliminated. For all the computations shown, the splitting by Ducros et al. is employed because a conservative splitting is more appropriate if one does not know if the subject flow is shock-free or has turbulence with shocks. Some attributes of the high-order filter approach are:

- Spatial base scheme: high-order and conservative, with high-order freestream preservation metric evaluation for curvilinear grids (no flux limiter or Riemann solver).

- Physical viscosity: automatically taken into consideration by the base scheme. The same order of central differencing for the viscous derivative, as the convective flux derivatives are used.

- Efficiency: one Riemann solve per dimension per time step, independent of time discretizations (less CPU time and fewer grid points than their standard shock-capturing scheme counterparts).

- Accuracy: containment of the numerical dissipation via local wavelet flow sensor.

- Well-balanced scheme: these nonlinear filter schemes are well-balanced schemes for certain chemical-reacting flows and problem-containing geometric source terms [17].

- Parallel algorithm: suitable for most of the current supercomputer architectures. 


\subsection{Sample test Cases Illustrating the Efficiency and Accuracy of High-Order Filter Schemes}

These filter schemes are efficient, and the total computational cost for a given error tolerance is lower than that for standard shock-capturing schemes of the same order. This is important, for example, in DNS and in flow control optimization, to improve aerodynamic properties, where the flow simulation must be carried out many times during the optimization loop. The efficiency and accuracy of the schemes for a wide variety of flow problems can be found in the aforementioned cited references. Here, two test cases are illustrated.

2-D Shock/Vorticity Interaction: Figure 1 shows a comparison of a second-order TVD, seventhorder weighted essentially nonoscillary (WENO) (WENO7), hybrid scheme (a switch between an eighth-order spatial central scheme and WENO7 with the use of a wavelet flow sensor as the switch indicator), and the filter scheme WENO7fi (an eighth-order spatial central base scheme and the dissipative portion of WENO7, and the same wavelet flow sensor, to guide where the WENO7 dissipation, should be applied at the postpossessing nonlinear filter step). A second-order Runge-Kutta method was used for the TVD scheme, and the classical fourth-order Runge Kutta method was used for the rest of the spatial scheme. For this particular simple 2-D shock/vorticity interaction test case with a simple weak planar shock without structure, WENO7, hybrid, and WENO7fi give the same accuracy. However, there is a large gain in CPU time by the filter scheme for this turbulence-free test case. For turbulence with shocks, there is more beneficial gain both in accuracy and CPU time of the filter schemes over their standard WENO counterparts.

1-D Shock/Turbulence Interaction Problem: This 1-D compressible inviscid ideal gas problem is one of the most computed test cases in the literature to assess the capability of a shockcapturing scheme in the presence of shock/turbulence interactions. The flow consists of a shock at Mach 3 propagating into a sinusoidal density field with initial data given by $\left(\rho_{L}, u_{L}, p_{L}\right)=$ $(3.857143,2.629369,10.33333)$ to the left of a shock located at $x=-4$ and $\left(\rho_{R}, u_{R}, p_{R}\right)=$ $(1+0.2 \sin (5 x), 0,1)$ to the right of the shock, where $\rho$ is the density, $u$ is the velocity, and $p$ is the pressure. The computational domain is $[-5,5]$, and the computation stops at time equal to 1.8 . Figure 2 shows the comparison between WENO3, WENO5, and WENO7, and their corresponding filter schemes WENO3fi, WENO5fi, and WENO7fi with the use of a very coarse uniform grid of 200 points with the reference solution. The reference solution is obtained with WENO5 using 16000 grid points. WENO5fi required, at the most, 50\% of the CPU time of WENO5 if the third or fourthorder Runge-Kutta time discretization was used. In order for WENO5 to obtain a similar accuracy

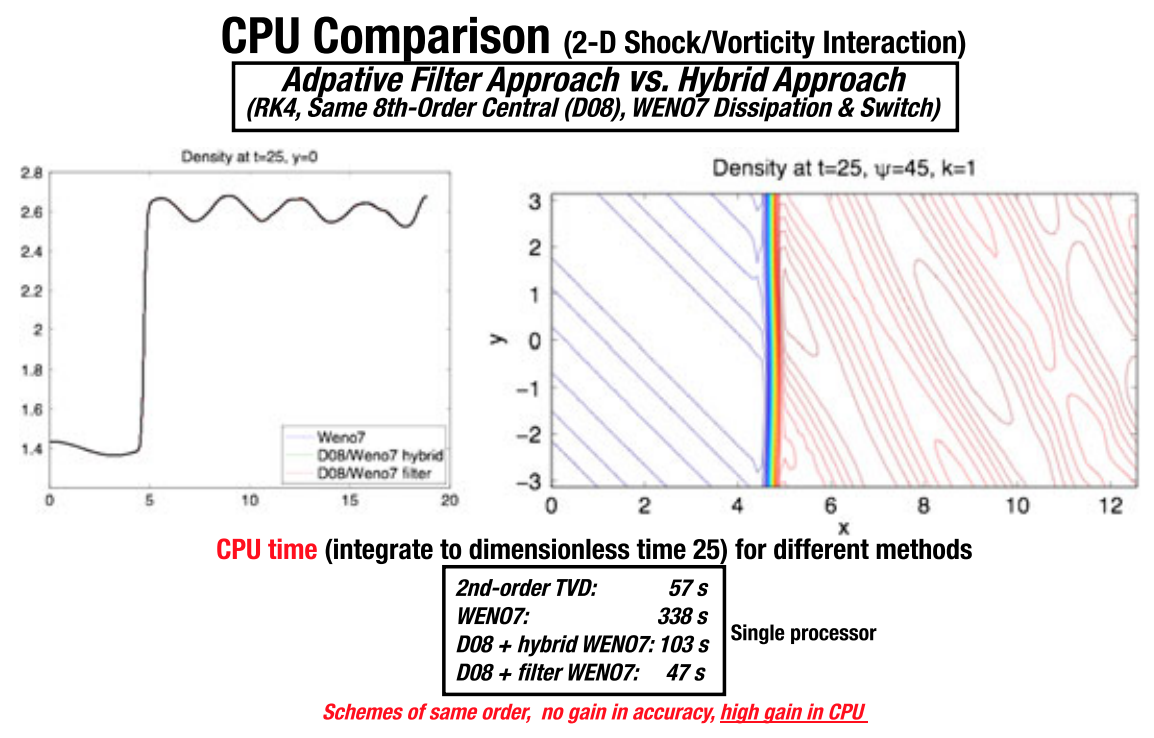

Figure 1. CPU comparison of four shock-capturing schemes. 


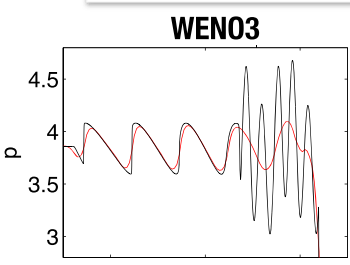

WEN05

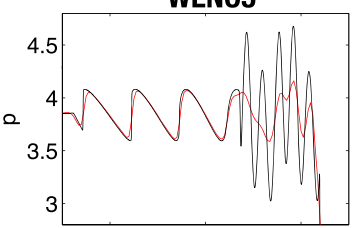

WEN07

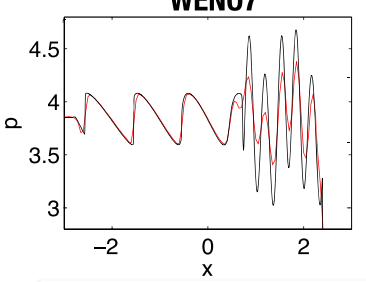

Accuracy of WEN05fi is similar to WEN09; less CPU

Figure 2. Same Numerical Dissipation. Nonlinear Filter + Flow Sensor vs. Built-in (1D Shock Turbulence Interaction: $\mathrm{M}=3$, Denslty, $200 \mathrm{pts}$ )

as that of WENO5fi, at least two times the number of grid points is needed. Moreover, the accuracy of WENO5fi is similar to that of WENO9 (computation not shown).

\subsection{Objective and Outline}

In this paper, we report recent studies of LES computations of compressible turbulent TML flows with the use of numerical schemes developed by Bell and Mehta, Bogdanoff, and Ducros et. al, $[5,7,12]$ in conjunction with the preprocessing step discussed previously. The current research is motivated by the overarching goal of developing numerical tools for reliable predictive capability of complex turbulent reacting flows, especially for problems including compressibility, heat transfer, and real gas effects interacting with instabilities, shocks, and turbulence. The comparative study between WENO7fi, WENO5, and WENO7 is reported in [18] with grid refinement studies was the first step in determining the suitable order of filter schemes to be used for the current physics-based study. The LES filtering issue in the presence of shocks [19] is not addressed. The paper is organized as follows: the numerical methods are given in Section 2. The subgrid models for the compressible Navier-Stokes equations are given briefly in Section 3 and Appendix A. Results are then presented and discussed in Section 4.

\section{NUMERICAL METHOD}

This section summarizes the numerical methods used in the turbulent TML study. The numerical methods solve the split form of the inviscid flux derivatives according to the preprocessing step. The discussion is broken up into two subsections. 


\subsection{Original high-order filter method}

For turbulence with shocks, instead of solely relying on very high-order high-resolution shockcapturing methods for accuracy, the filter schemes $[5,12,14,20,21]$ take advantage of the effectiveness of the nonlinear dissipation contained in good shock-capturing schemes, as stabilizing mechanisms at locations where needed. Such a filter method consists of two steps: a full time step using a spatially high-order nondissipative base scheme, followed by a postprocessing filter step. The postprocessing filter step consists of the products of wavelet-based flow sensors and nonlinear numerical dissipations. The flow sensor is used in an adaptive procedure to analyze the computed flow data and indicate the location and the type of built-in numerical dissipation that can be eliminated or further reduced. The nonlinear dissipative portion of a high-resolution shock-capturing scheme can be any TVD, MUSCL, ENO, or WENO scheme. By design, the flow sensors, spatial base schemes, and nonlinear dissipation models are stand-alone modules. Therefore, a whole class of low dissipative high-order schemes can be derived with ease. Unlike standard shock-capturing and/or hybrid shock-capturing methods, the nonlinear filter method requires one Riemann solve per dimension independent of time discretizations. The nonlinear filter method is more efficient than its shock-capturing method counterparts employing the same order of respective methods.

Recently, these filter schemes were proven to be well-balanced schemes [17], in the sense that these schemes preserve exactly the certain steady state solutions of the chemical nonequilibrium governing equation. With this added property, these filter schemes can better minimize spurious numerics in reacting flows containing mixed steady shocks and unsteady turbulence with shocklet components than standard nonwell-balanced shock-capturing schemes. In addition, for some stiff reacting flow test cases, the high-order filter scheme is able to obtain the correct propagation speed of discontinuities, whereas the standard high-order WENO scheme cannot [22,23].

For simplicity of the presentation, the discussion for the base scheme and postprocessing step of the filter scheme is restricted to the inviscid part of the Navier-Stokes equations. For viscous gas dynamics, the same order of spatial-centered base scheme for the convection terms and the viscous terms are employed. For all of the LES computations, the classical fourth-order Runge-Kutta time discretization is employed.

Consider the 3-D compressible Euler equations in Cartesian geometry,

$$
U_{t}+\nabla \cdot \mathbf{F}=\mathbf{0} ; U=\left(\begin{array}{c}
\rho \\
\mathbf{m} \\
e
\end{array}\right) ; \mathbf{F}=\left(\begin{array}{c}
\rho \mathbf{u} \\
\rho \mathbf{u} \mathbf{u}^{T}+p \\
\mathbf{u}(e+p)
\end{array}\right)
$$

Here, the velocity vector $\mathbf{u}=(u, v, w)^{T}$, the momentum vector $\mathbf{m}=(\rho u, \rho v, \rho w), \rho$ is the density, and $e$ is the total energy.

In a Cartesian grid, denote the grid indices in three spatial direction as $(j, k, l)$. The spatial base scheme used to approximate the $x$ inviscid flux derivatives, $F(U)_{x}$, (with the grid indices $k$ and $l$ for the $y$-directions and $z$-directions suppressed) is written as, for example,

$$
\frac{\partial F}{\partial x} \approx D_{08} F_{j}
$$

where $D_{08}$ is the standard eighth-order accurate centered difference operator. See [16] for the split form of Equation (2).

After the completion of a full Runge-Kutta time step of the base scheme step, the second step is to adaptively apply a postprocessing nonlinear filter. The nonlinear filter can be obtained, for example in the $x$-direction, by taking the full seventh-order WENO scheme (WENO7) [24] for the inviscid flux derivative in the $x$-direction and subtracting $D_{08} F_{j}$. The final update of the solution is (with the numerical fluxes in the $y$-directions and $z$-directions suppressed, as well as their corresponding $y$-directions and $z$-directions indices on the $x$ inviscid flux)

$$
U_{j, k, l}^{n+1}=U_{j, k, l}^{*}-\frac{\Delta t}{\Delta x}\left[H_{j+1 / 2}^{*}-H_{j-1 / 2}^{*}\right] .
$$


The nonlinear filter numerical fluxes usually involve the use of field-by-field approximate Riemann solvers. If the Roe type of approximate Riemann solver [25] is employed for example, the $x$-filter numerical flux vector $H_{j+1 / 2}$ evaluated at the $U^{*}$ solution from the base scheme step is

$$
H_{j+1 / 2}=R_{j+1 / 2} \bar{H}_{j+1 / 2},
$$

where $R_{j+1 / 2}$ is the matrix of the right eigenvectors of the inviscid flux vector's Jacobian, in terms of the Roe's average states. Denote the elements of the vector $\bar{H}_{j+1 / 2}$ by $\bar{h}_{j+1 / 2}^{l}, l=1,2, \ldots, 5$. The nonlinear portion of the filter $\bar{h}_{j+1 / 2}^{l}$ has the form

$$
\bar{h}_{j+1 / 2}^{l}=\frac{\kappa}{2} \omega_{j+1 / 2}^{l} \phi_{j+1 / 2}^{l} .
$$

Here, $\omega_{j+1 / 2}^{l}$ is the wavelet flow sensor used to activate the nonlinear numerical dissipation $\frac{1}{2} \phi_{j+1 / 2}^{l}$, and the original formulation for $\kappa$ is a positive parameter that is less than or equal to one. Some tuning of the parameter $\kappa$ is needed for different flow types. A local $\kappa$, to be discussed next, depending on the local Mach number for low speed flows and depending on local shock strength for high-speed flows, would minimize the tuning of parameters. A local flow sensor was studied by Lo et al. [26] who took advantage of the Ducros et al. shock flow sensor [27] to obtain a local artificial compression method (ACM) sensor for the original filter scheme of Yee et al. [20].

The dissipative portion of the nonlinear filter $\frac{1}{2} \phi_{j+1 / 2}^{l}=g_{j+1 / 2}^{l}-b_{j+1 / 2}^{l}$ is the dissipative portion of, for example, WENO7 for the local $l$ th-characteristic wave. Here, $g_{j+1 / 2}^{l}$ and $b_{j+1 / 2}^{l}$ are numerical fluxes of WENO7 and the eighth-order central scheme for the $l$ th characteristic, respectively. Hereafter, we denote this filter scheme as WENO7fi.

A summary of the three basic steps used to obtain $\omega_{j+1 / 2}^{l}$ can be found in $[5,12]$. For example, the flow sensor $\omega_{j+1 / 2}^{l}$, which was utilized to activate the shock-capturing dissipation with the use of the cut off procedure, is a vector (if applied dimension-by-dimension) consisting of ' 1 's' and '0's'. For all of the computations, a three-level second-order Harten multiresolution wavelet decomposition of the computed density and pressure is used as the flow sensor [12].

\subsection{Improved high-order filter method}

Previous numerical experiments on a wide range of flow conditions $[5,12,14,20,21]$ indicated that the original filter scheme improves the overall accuracy of the computation compared with standard shock-capturing schemes of the same order. Studies found that the improved accuracy is more pronounced if the parameter $\kappa$ in Equation (4) is tuned according to the flow type locally. For hypersonic flows with strong shocks, $\kappa$ is set to 1 . For high subsonic and supersonic flows with strong shocks, $\kappa$ is in the range of $(0.3,0.9)$. For low speed turbulent flows without shocks or long time integration of smooth flows, $\kappa$ can be one to two orders of magnitude smaller than 1 . In other words, $\kappa$ should be the flow location, shock strength, and local flow-type dependent. The improved $\kappa$ proposed in [7] consists of a simple global $\kappa$ for smooth flows and a local $\kappa$ for problems with shocks and turbulence.

2.2.1. An efficient global $\kappa$ for low Mach number and smooth flows. The flow speed indicator formula by Li and Gu used to overcome the shortcomings of the 'low speed Roe scheme' [28] was modified to obtain an improved global $\kappa$ denoted by $\bar{\kappa}$ for Equation (4) to minimize the tuning of the original $\kappa$ for low Mach number flows. $\bar{\kappa}$ has the form

$$
\bar{\kappa}=f_{1}(M) \kappa,
$$

with

$$
f_{1}(M)=\min \left(\frac{M^{2}}{2} \frac{\sqrt{4+\left(1-M^{2}\right)^{2}}}{1+M^{2}}, 1\right)
$$


Here, $M$ is the maximum Mach number of the entire computational domain at each stage of the time evolution. $f_{1}(M)$ has the same form as [28] except there is an extra factor ' $\frac{M}{2}$ ' added to the first argument on the right-hand side of the original form $f(M)$ in [28, Equation (18)]. The added factor provides a similar value of the tuning $\kappa$ observed in the numerical experimentations reported in the aforementioned cited references. With the flow speed indicator $f_{1}(M)$ in front of $\kappa$, the same $\kappa$ used for the supersonic shock problem can be used without any tuning for the very low speed turbulent flow cases. Another minor modification of the above is

$$
\overline{f_{1}(M)}=\max \left(\min \left(\frac{M^{2}}{2} \frac{\sqrt{4+\left(1-M^{2}\right)^{2}}}{1+M^{2}}, 1\right), \epsilon\right),
$$

where $\epsilon$ is a small threshold value used to avoid completely switching off the dissipation. A function that retains the majority of $f_{1}(M)$, but includes larger Mach number for not very strong shocks, is

$$
f_{2}(M)=(Q(M, 2)+Q(M, 3.5)) / 2
$$

or

$$
\overline{f_{2}(M)}=\max ((Q(M, 2)+Q(M, 3.5)) / 2, \epsilon),
$$

where

$$
Q(M, a)=\left\{\begin{array}{ll}
P(M / a) & M<a \\
1 & \text { otherwise }
\end{array} .\right.
$$

The polynomial

$$
P(x)=x^{4}\left(35-84 x+70 x^{2}-20 x^{3}\right)
$$

is monotonically increasing from $P(0)=0$ to $P(1)=1$ and has the property $P^{\prime}(x)$, which has three continuous derivatives at $x=0$ and at $x=1$.

Below supersonic speeds, a simple and efficient global $\bar{\kappa}$ can be obtained according to the maximum Mach number of the entire flow field and, the value is determined by $f_{1}(M)$ or $f_{2}(M)$ for nonzero $\omega_{j+1 / 2}^{l}$. It is noted that if the original $f(M)$ was used, instead of $f_{1}(M)$ or $f_{2}(M)$ in Equation (5), the amount of nonlinear filter dissipation could be too large for very low speed turbulent flows (for the same fixed $\kappa$ ). See Figure 3 for details.

2.2.2. Local flow sensor for a wide spectrum of flow speed and shock strength. At each time step and grid point, the aforementioned global $\bar{\kappa}$ is not sufficient to reduce the amount of numerical dissipation where needed for flows that contain a variety of flow features. A more appropriate approach

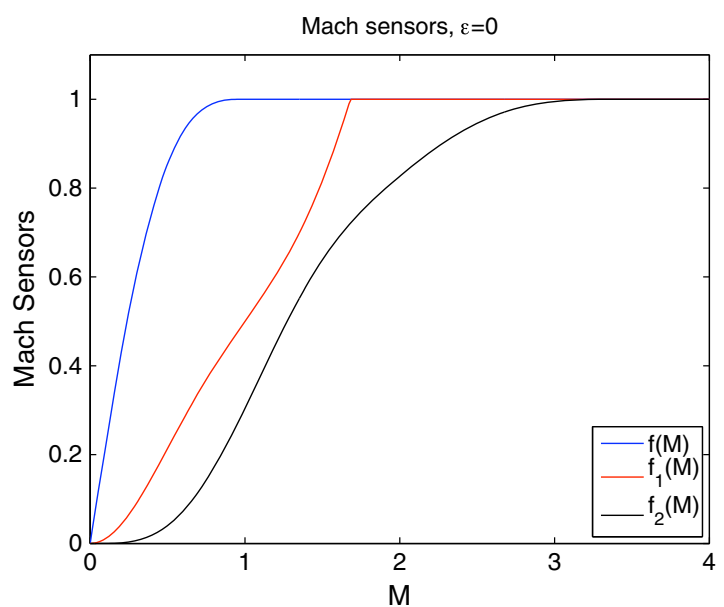

Figure 3. Mach number sensors: $f(M)$ (blue) function by Li and Gu, $f_{1}(M)$ (red) the modified $f(M)$, and $f_{2}(M)$ (black) (includes low supersonic Mach numbers). 
is to obtain a 'local $\kappa^{\prime}$, which is determined according to the above at each grid point. If known, a dominating shock jump variable should be used for shock detections. In other words, the filter numerical flux indicated in Equation (4) is replaced by

$$
\bar{h}_{j+1 / 2}^{l}=\frac{1}{2}\left[\kappa_{j+1 / 2}^{l} \omega_{j+1 / 2}^{l} \phi_{j+1 / 2}^{l}\right] .
$$

In the case of unknown physics and without experimental data or theory for comparison, $\kappa_{j+1 / 2}^{l}$ has to depend on the local Mach number in the low speed or smooth flow regions, on the local shock strength in shock regions, and on the turbulent fluctuations in vortical regions in order to minimize the tuning of parameters. According to the local flow type, for each nonzero wavelet indicator, $\omega_{j+1 / 2}^{l}, \kappa_{j+1 / 2}^{l}$ should provide the aforementioned amount (between $(0,1)$ ) to be filtered by the shock-capturing dissipation $\phi_{j+1 / 2}^{l}$. For problems containing turbulence and strong shocks, the shock strength should come into play. One measure of the shock strength can be based on the numerical Schlieren formula [29] for the chosen variables that exhibit the strongest shock strength. In the vicinity of turbulent fluctuation locations, $\kappa_{j+1 / 2}^{l}$ will be kept in the same order as in the nearly incompressible case, except in the vicinity of high shear and shocklets.

Because $\bar{\kappa}$ works well for local Mach number below $0.4, \kappa$ only needs to be modified in regions that are above 0.4. In other words, the final value of $\kappa_{j+1 / 2}^{l}$ is determined by the previous local $\bar{\kappa}$ if the local Mach number is below 0.4. If the local Mach number is above 0.4 at discontinuities detected by the nonzero wavelet indicator, $\omega_{j+1 / 2}^{l}, \kappa_{j+1 / 2}^{l}$ is determined by the shock strength (normalized between $(0,1)$ ) on the basis of the Schlieren formula near discontinuities. Again, if known, dominating shock jump variables should be used for shock detections. At locations with turbulence, determined by the turbulent sensor (e.g., $\omega_{j+1 / 2}^{l}$ obtained from employing wavelets with higher order vanishing moments), $\kappa_{j+1 / 2}^{l}$ is kept in the same order, as in the nearly incompressible case, except in the vicinity of high shear and shocklet locations where a slightly larger $\kappa_{j+1 / 2}^{l}$ would be used. Methods in detecting turbulent flow can be (i) wavelets with higher order vanishing moments, and (ii) wavelet-based coherent vortex extraction (CVE) by Farge et al. [30] (Split the flow into two parts: active coherent vortices and incoherent background flow).

Results by the local $\kappa_{j+1 / 2}^{l}$ that take the local flow speed and shock strength into consideration will be reported in [11], an expanded version of [7]. A preliminary study with more complex shock turbulence problems and the applicability of even wider flow types indicates the necessity of the local $\kappa_{j+1 / 2}^{l}$.

\section{LES IMPLEMENTATION}

In terms of turbulence modeling, there has been considerable progress in the development and usage of LES for the simulation of turbulent flows in the past few decades. This has been facilitated by the substantial increase in computing power. The triumphant journey of LES started with the pioneering work of Smagorinsky [31], Lilly [32], Deardoff [33], Germano [34] and others. Comprehensive accounts on LES are provided by Sagaut [35] and Pope [36,37], and reviews at different stages of the development are provided by Rogallo and Moin [38], Galperin and Orszag [39], Lesieur and Metais [40], and Meneveau and Katz [41]. The LES model used for the current simulation is discussed here with subgrid model summaries in Appendix A.

In LES, the large-scale field can be obtained from the solution of the filtered Navier-Stokes equations, whereas scales smaller than the grid size are modeled. The filtering operation, which defines the large-scale variables (denoted by an overbar), can be written as

$$
\bar{\varphi}(\vec{x})=\int_{D} G(\vec{x}-\vec{y}, \Delta) \varphi(\vec{y}) d \vec{y},
$$

where $D$ is the flow domain, $G$ is the filter function, and $\Delta$ is the filter width associated with the wavelength of the smallest scale retained by the filtering operation. 
For compressible flows, in order to avoid unclosed sulgrid scale (SGS) terms in the continuity equation, a density-weighted Favre filter operator is introduced. This operator is defined as $\widetilde{\varphi}=\overline{\rho \varphi} / \bar{\rho}$.

Favre-filtered continuity, momentum, and total energy equations in conservative form are, respectively,

$$
\begin{gathered}
\frac{\partial \bar{\rho}}{\partial t}+\frac{\partial \bar{\rho} \widetilde{u}_{i}}{\partial x_{i}}=0 \\
\frac{\partial\left(\bar{\rho} \widetilde{u}_{i}\right)}{\partial t}+\frac{\partial\left(\bar{\rho} \widetilde{u}_{i} \widetilde{u}_{j}\right)}{\partial x_{j}}+\frac{\partial \bar{p}}{\partial x_{i}}=\frac{\partial \breve{\sigma}_{i j}}{\partial x_{j}}-\frac{\partial \tau_{i j}}{\partial x_{j}} \\
\frac{\partial(\bar{\rho} \breve{E})}{\partial t}+\frac{\partial}{\partial x_{j}}\left[(\bar{\rho} \breve{E}+\bar{p}) \widetilde{u}_{j}-\breve{\sigma}_{i j} \widetilde{u}_{i}+\breve{q}_{j}\right]=-\frac{\partial}{\partial x_{j}}\left(\mathcal{Q}_{j}-\mathcal{D}_{j}^{v}-\mathcal{J}_{j}+\frac{1}{2} \mathcal{E}_{v}\right) .
\end{gathered}
$$

Unlike the 'bar' and the 'tilde', the 'breve' symbol does not denote a filter operation but indicates that the quantity is based on primitive filtered variables. Thus, $\breve{E}$ refers to the resolved total energy, which is not equal to the filtered total energy. The resolved viscous stress tensor $\breve{\sigma}_{i j}$ and the heat flux are defined as

$$
\breve{\sigma}_{i j}=2 \mu(\widetilde{T})\left(\widetilde{S}_{i j}-\frac{1}{3} \delta_{i j} \partial_{k} \widetilde{u}_{k}\right) \quad \text { with } \quad \widetilde{S}_{i j}=\left(\partial_{j} \widetilde{u}_{i}+\partial_{i} \widetilde{u}_{j}\right) / 2
$$

and

$$
\breve{q}_{j}=-\lambda(\widetilde{T}) \frac{\partial \widetilde{T}}{\partial x_{j}}
$$

where $\mu(\widetilde{T})$ and $\lambda(\widetilde{T})$ are the viscosity and thermal conductivity corresponding to the filtered temperature $\widetilde{T}$. The constitutive equations are

$$
\bar{\rho} \breve{E}=\frac{\bar{p}}{\gamma-1}+\frac{1}{2} \bar{\rho} \widetilde{u_{j}} \widetilde{u_{j}} \quad \text { and } \quad \bar{p}=c_{v}(\gamma-1) \bar{\rho} \widetilde{T},
$$

where the heat capacity at constant volume, $c_{v}$, and the ratio of heat capacities, $\gamma$, are given constants. The subgrid-scale stress is defined as

$$
\tau_{i j}=\bar{\rho}\left(\widetilde{u_{i} u_{j}}-\widetilde{u}_{i} \widetilde{u}_{j}\right)
$$

and the subgrid terms of the energy equation are

$$
\begin{aligned}
\mathcal{Q}_{j} & =\bar{\rho} \gamma c_{v}\left(\widetilde{u_{j} T}-\widetilde{u}_{j} \widetilde{T}\right) \\
\mathcal{J}_{j} & =\bar{q}_{j}-\breve{q}_{j} \\
\mathcal{D}_{j}^{v} & =\overline{\sigma_{j k} u_{k}}-\widetilde{\sigma_{j k}} \widetilde{u_{k}} \\
\mathcal{E}_{j} & =\bar{\rho}\left(\widetilde{u_{j} u_{k} u_{k}}-\widetilde{u_{j}} \widetilde{u_{k} u_{k}}\right) .
\end{aligned}
$$

We will model the subgrid terms in order to close the system. The model for the subgrid stress, $\tau_{i j}$, employs the eddy viscosity hypothesis with an eddy viscosity, $\mu_{t}$, having either a simple Smagorinsky model or a more advanced dynamic model. The details are given in Appendix A. In the energy equation, the subgrid terms $\mathcal{J}_{j}, \mathcal{D}_{j}^{v}$, and $\mathcal{E}_{j}$ are set as equal to zero, and the subgrid-scale heat flux is modeled using the eddy diffusivity hypothesis,

$$
\mathcal{Q}_{j}=-\frac{\mu_{t} \gamma c_{v}}{P r_{t}} \frac{\partial \widetilde{T}}{\partial x_{j}}
$$

where the turbulent Prandtl number, $P r_{t}$, is a given constant. 


\section{LES OF TEMPORALLY EVOLVING COMPRESSIBLE MIXING LAYERS (TML)}

In this section, employing WENO7fi, we consider the LES of temporally evolving mixing layers between two streams moving with opposite velocities, with $U_{1}=-U_{2}=\Delta U / 2$. From here on, WENO7fi refers to the preprocessing step (Ducros et al. splitting of the inviscid flux derivative) in conjunction with the eighth-order central spatial base scheme with the dissipative portion of WENO7 and the global flow sensor discussed in Section 2 with $\kappa=0.7$. The three main characteristics of compressible mixing layers are (i) the self-similarity property, which is characterized by the linear growth of the layer width as well as the mean velocities and turbulent statistics being independent of the downstream distance when normalized by the appropriate length and velocity scales; (ii) the compressibility effects through turbulence damping and decrease of the mixing-layer growth rate for high convective Mach numbers; and (iii) the presence of large-scale structures with shocklets. These organized structures play an important role in the dynamics of the mixing layer, its spreading, and energy transport. The objective of the current investigation is to verify that the WENO7fi used in this study is capable of capturing the three key points cited above.

\subsection{Problem setup}

The configuration of the TML is shown in Figure 4. Five test cases (denoted LES-C $C_{i}, i=1, \ldots, 5$ ) are carried out with different convective Mach numbers $\left(M_{c}\right)$ ranging from the incompressible case $M_{c}=0.1$ up to the supersonic case $M_{c}=1.5$. The latter corresponds to highly compressible mixing layers, whereas the first two cases $M_{c}=0.1$ and $M_{c}=0.3$ can be considered as quasiincompressible and are compared with the experimental results of an incompressible shear layer. All of the simulations described are performed at an initial Reynolds number, $R e_{\omega_{0}}$, on the basis of the mean velocity difference $\Delta U$, the average viscosity of the free streams, and the vorticity thickness $\delta_{\omega_{0}}$ of 800 with $\delta_{w_{0}}=4 \delta_{\theta_{0}}$, where $\delta_{\omega}=\Delta U /\langle\partial u / \partial y\rangle_{\max }$ is the vorticity thickness of the shear layer, and $\delta_{\theta}$ is the momentum thickness given in Equation 17 (see later section). It is worth noticing that $R e_{\omega}$ reaches values as large as $3 \times 10^{5}$ at the end of the simulation, which is one order of magnitude higher than similar DNS and LES computations reported in the literature [42-44]. Table I summarizes the details of the flow parameters for both LES and previous DNS data of the literature. The mean flow is initialized with a tangent hyperbolic profile for the streamwise velocity, $u(y)=\frac{1}{2} \Delta U \tanh \left[y /\left(2 \delta_{\theta_{0}}\right)\right]$, whereas the two other velocity components are set to zero. In addition to these mean values, three-dimensional turbulent fluctuations $\left(u^{\prime}, v^{\prime}, w^{\prime}\right)$ are imposed, whereas initial pressure and density are set as constants. Because the simulation is temporal, the initial velocity perturbations are computed using a digital filter technique [45]. This procedure utilizes the prescribed Reynolds stress tensor and length scales of the problem concerned to generate the corresponding fluctuating velocity field, taking into account the nature of the autocorrelation function for the prevailing turbulence. The digital filter algorithm is given in Appendix B. The length scales are chosen as $\delta_{w_{0}}$ in each direction. The Reynolds stresses have a Gaussian shape in $y$, with

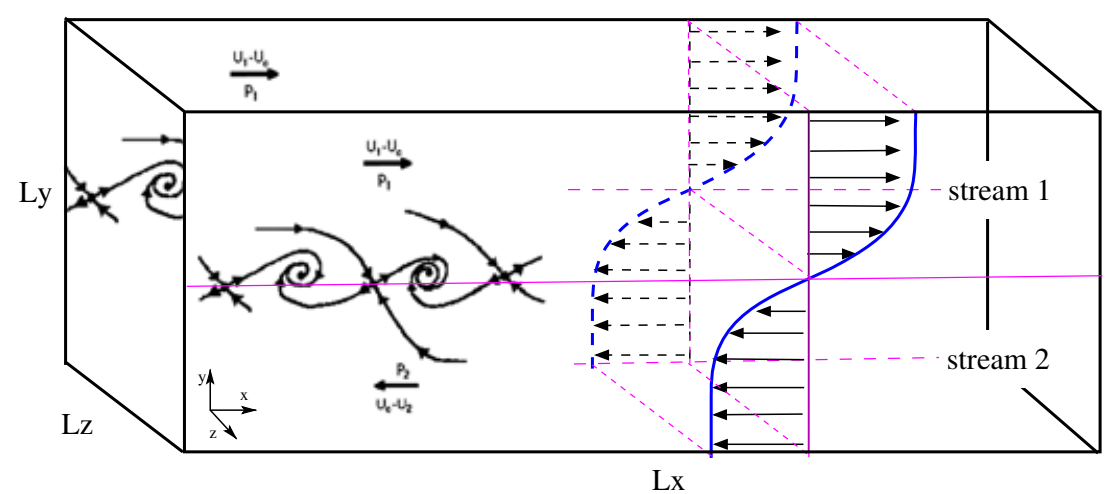

Figure 4. Schematic configuration of the temporal mixing layer.

Published 2012. This article is a US Government work and is in the public domain in the USA.
Int. J. Numer. Meth. Fluids 2012; 70:1405-1427

DOI: $10.1002 /$ fld 
Table I. Flow parameters and turbulent length scales during the quasi-self-similar stage.

\begin{tabular}{llccccc}
\hline Case & $M_{c}$ & $R e_{\omega_{0}}$ & $L_{x} / \Lambda_{x}$ & $L_{z} / \Lambda_{z}$ & $\Delta y_{\text {min }} / \lambda$ & $\Delta y_{\text {min }} / \eta$ \\
\hline DNS [48] & 0 & 800 & - & - & & \\
DNS [44] & 0.3 & 640 & 20.4 & 15.3 & $>1$ & $\approx 1$ \\
LES-C1 & 0.1 & 800 & 30.2 & 15.1 & 0.64 & 49.3 \\
LES-C2 & 0.3 & 800 & 30.2 & 15.1 & 0.65 & 49.5 \\
LES-C3 & 0.8 & 800 & 12.1 & 11.0 & 0.74 & 50.1 \\
LES-C4 & 1.0 & 800 & 12.0 & 11.4 & 0.67 & 53.7 \\
LES-C5 & 1.5 & 800 & 12.0 & 10.2 & 0.82 & 55.2 \\
\hline
\end{tabular}

amplitudes chosen to be similar to the experimental peak intensities observed in the incompressible mixing layer [46].

Periodic boundary conditions are enforced in the streamwise $(x)$ and spanwise $(z)$ directions, whereas nonreflecting conditions are applied at both the top and bottom boundaries ( $y$-direction). The use of a periodic boundary condition in the $x$-direction corresponds to the temporal formulation of mixing-layer evolution, which evolves only over time as it spreads in $y$.

\subsection{Mesh requirements}

Similar to [42], a computational domain of lengths $L_{x} \times L_{y} \times L_{z}=1200 \delta_{\theta_{0}} \times 370 \delta_{\theta_{0}} \times 270 \delta_{\theta_{0}}$ is used with the corresponding mesh points $N_{x} \times N_{y} \times N_{z}=512 \times 211 \times 131$. The same grid system uniformly spaced in the $x$-directions and $z$-directions, and stretched in the $y$-direction is employed for all considered cases. The stretching function for the $y$-direction is based on $\frac{L_{y}}{2} \frac{\sinh \left(b_{y} \eta\right)}{\sinh b_{y}}$, where $L_{y}$ is the box size in the $y$-direction and the stretching factor $b_{y}=3.4$. The mapped coordinate $\eta$ is equally spaced and runs from -1 to 1 . The high resolution (HR) grid used in this study contains an order of fewer magnitude cells than that of the DNS of Pantano and Sarkar [44] compared to the domain length. The emphasis of the HR simulation is to produce an LES solution that predicts the trends of the DNS, as well as experimental data for both quasi-incompressible and highly compressible mixing layers. To ensure that the computational domain in the $x$-directions and $z$-directions is sufficiently wide, we analyzed the two-point correlation functions

$$
R_{\varphi \varphi}(r)=\sum_{k=1}^{N-k_{r}} \overline{\varphi_{k}^{\prime} \varphi_{k+k_{r}}^{\prime}}, \quad k_{r}=0,1, \ldots, N-1,
$$

where $r=k_{r} \Delta, N$ is half of the number of grid points in the homogeneous directions with grid size $\Delta$, and $\varphi^{\prime}$ represents the fluctuations of flow variables.

The computed two-point autocorrelation coefficients $R_{\varphi \varphi}(r) / R_{\varphi \varphi}(0)$ (pressure as well as velocity components) in the homogeneous directions ( $x$ and $z$ ) are reported in Figure 5 as a function of the distance in the streamwise and spanwise coordinates at the middle of the mixing layer $\left(L_{y} / 2\right)$. This figure shows that the flow variables are sufficiently decorrelated over distances $L_{x} / 2$ and $L_{z} / 2$, thus ensuring that the streamwise, as well as the spanwise extents of the computational domain, is sufficient in order to not inhibit turbulence dynamics. Also, the length in the $y$-direction is selected to be large enough for the flow to achieve a fully developed state before the effects of the upper and lower boundaries are felt. In terms of turbulent length scales, the Kolmogorov length scale $\eta$ and an average (isotropic) Taylor microscale $\lambda$ are defined as $\eta=\left(v^{3} / \varepsilon\right)^{1 / 4}$ and $\lambda=\sqrt{15 v k / \varepsilon}$, where $k=\frac{1}{2}\left(\overline{u^{\prime 2}}+\overline{v^{\prime 2}}+\overline{w^{\prime 2}}\right)$ is the turbulent kinetic energy. The computed integral length scales $\left(\Lambda_{x}, \Lambda_{z}\right)$ and the Kolmogorov scale are also given in Table I for further comparison. In our case, the integral scales are given by

$$
\Lambda_{x}=\int_{0}^{L_{x} / 2} \frac{\left\langle u_{i}\left(x_{i}, t\right) u_{i}\left(x_{i}+p, t\right)\right\rangle}{\left\langle u_{i}^{2}\right\rangle} d p, \quad \Lambda_{z}=\int_{0}^{L_{z} / 2} \frac{\left\langle u_{i}\left(x_{i}, t\right) u_{i}\left(x_{i}+p, t\right)\right\rangle}{\left\langle u_{i}^{2}\right\rangle} d p,
$$

where $p$ is the distance between two points in the flow. The integral length scale is important in characterizing the structure of turbulence. It is a measure of the longest correlation distance between the 

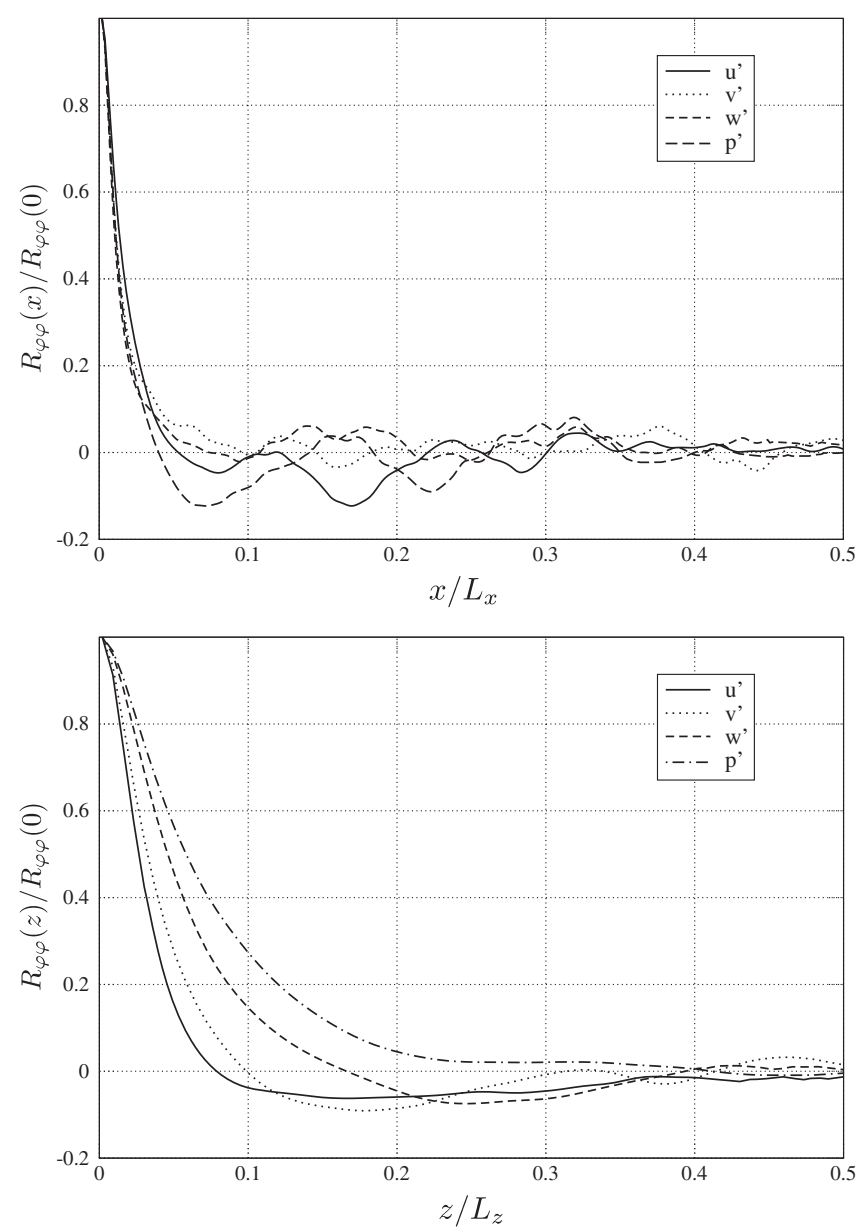

Figure 5. Streamwise and spanwise autocorrelation functions for LES-C5.

flow velocity (or vorticity, etc) at two points in the flow field. Recent work concludes that a reasonable lower limit of the domain must be at least six times larger than the integral length [47]. This recommendation is consistent with the data shown in Table I, where the spatial domain is between ten and thirty times larger than the integral length. Also, it is evident from Table I that the integral lengths are sufficiently small compared to the computational, domain and the grid resolution is adequate to resolve the large scales of turbulence.

Because of the high computational cost of the simulations, the numerical code is fully parallelized running on up to 600 processors. In total, the present simulation required $2000 \mathrm{CPU}$ hours each on modern SGI IC, Pleiades, and Columbia supercomputers at NASA Ames Research Center.

\subsection{Mean flow and turbulent statistics}

LES computations are carried out up to dimensionless time $\tau=t \Delta U / \delta_{\theta_{0}} \simeq 3000$ for the higher convective Mach number cases and $\tau \simeq 1200$ for the quasi-incompressible cases. In order to compare the LES results with experimental data, the time-averaged flow quantities $\langle\bar{\varphi}\rangle$ and $\langle\widetilde{\varphi}\rangle$ are extracted from the flow field during the self-similar time period $(600<\tau<1000$ for LES-C1 and LES-C2, and $2000<\tau<2800$ for LES-C3, LES-C4, and LES-C5). Note that throughout this paper only resolved quantities are considered; subgrid-scale contributions are not added onto, for example, the turbulent stresses. For the validation of the low-Mach-number LES case, previous DNS studies of the incompressible shear layer, including Rogers and Moser [48], and Pantano and Sarkar [44], as well as experimental studies by Bell and Mehta [46], and Spencer and Jones [49], are used. Further 


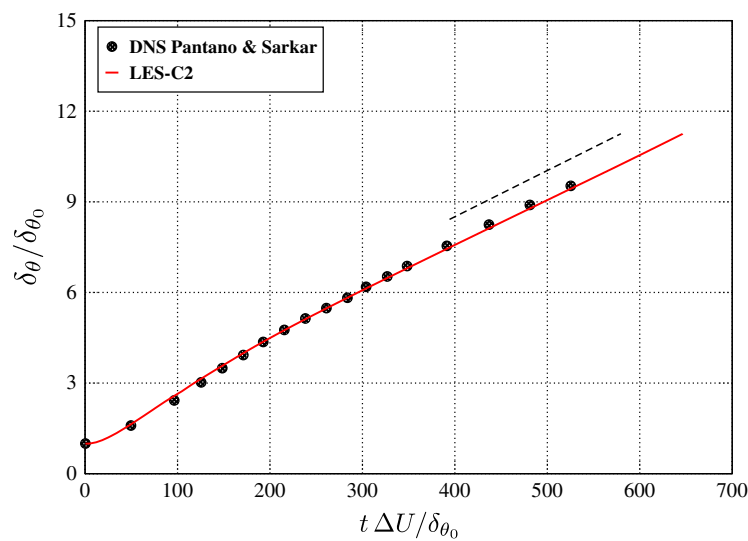

Figure 6. Time evolution of normalized momentum thickness for LES-C2 compared to the DNS of Pantano and Sarkar [44] for $M_{c}=0.3$.

experimental results on the compressible shear layer (Papamoschou and Roshko [50], Elliot and Samimy [51], Barre et al. [52], and Chambres et al. [53]) and DNS results obtained by Pantano and Sarkar [44] are used to compare with the high-Mach number simulations.

As recommended by Rogers and Moser [48], the momentum thickness $\delta_{\theta}$ is used for self-similar scaling rather than the vorticity thickness $\delta_{\omega}$. This is because it is less sensitive to statistical noise as it is an integral quantity evolving smoothly in time, whereas $\delta_{\omega}$ is obtained from the derivative of the mean velocity and may exhibit oscillations during the flow evolution. Therefore, the time evolution of the momentum thickness of the flow calculated using the definition

$$
\delta_{\theta}=\int_{-\infty}^{+\infty} \frac{\bar{\rho}}{\rho_{\text {ref }}}\left(\frac{1}{4}-\frac{\widetilde{u}^{2}}{\Delta U^{2}}\right) d y
$$

is shown in Figure 6. An excellent agreement with the DNS simulation is obtained, and the linear slope is recovered after a short transient, showing the self-similar state of the mixing layer. The growth rate is $d\left(\delta_{\theta} / \delta_{\theta_{0}}\right) / d \tau=0.016$ (slope of the linear curve fit). The ratio of vorticity thickness to momentum thickness is $D_{\omega}=\delta_{\omega} / \delta_{\theta} \simeq 4.5$ with $R e_{\omega}=603391$ at $\tau_{\max } \simeq 1200$. This is in excellent agreement with the DNS growth rate of the quasi-incompressible case with $M_{c}=0.3$ by Pantano and Sarkar [44]. Note that in Equation 17, () ref represents the reference state which is the arithmetic mean of the free stream 1 and 2.

The normalized mean streamwise velocity for LES-C1 and LES-C2 are presented in Figure 7. It can be seen that the two LES profiles collapse together and are in excellent agreement with both DNS [44] and experimental results [46,49].

Further validation of the present LES is achieved through the comparison of turbulent intensities in the self-similar region (calculated by averaging over profiles plotted in similar coordinates) seen in Figure 8, where different components of the normalized Reynolds stress tensor, $\sqrt{R_{i j}} / \Delta U$ $\left(R_{i j}=\overline{\rho u_{i}^{\prime \prime} u_{i}^{\prime \prime}} / \bar{\rho}\right)$, are compared to the DNS and experimental data. Interestingly, at $M c=0.1$, the LES agrees better with the experimental $[46,49]$ and DNS [48] data in the incompressible shear layer than the DNS results of Pantano and Sarkar [44]. Table II summarizes the comparison of peak turbulent intensities, as well as the anisotropic deviation on the centerline of the layer. It is evident that a very good agreement between the present LES and previous results is obtained for this measure of anisotropy. It is important to notice that both LES-C1 and LES-C2 give almost the same results, probably because both are in the incompressible (or weakly-compressible) regime.

\subsection{Compressibility effects}

Apart from studying the self-similarity property in the mixing layer, we also investigated the effects of the convective Mach number by using LES. Figure 9 shows the time evolution of the momentum thickness for various convective Mach numbers. It can be seen that after a relatively long 


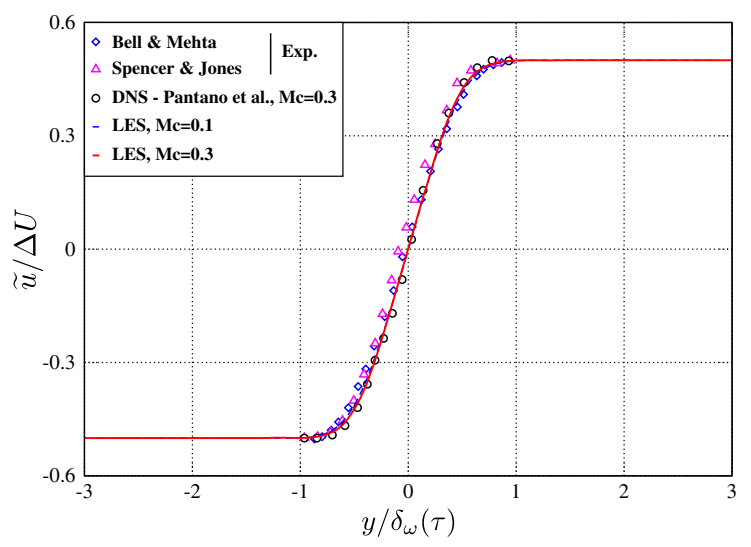

Figure 7. Comparison of normalized mean streamwise velocity between LES-C1 and LES-C2.
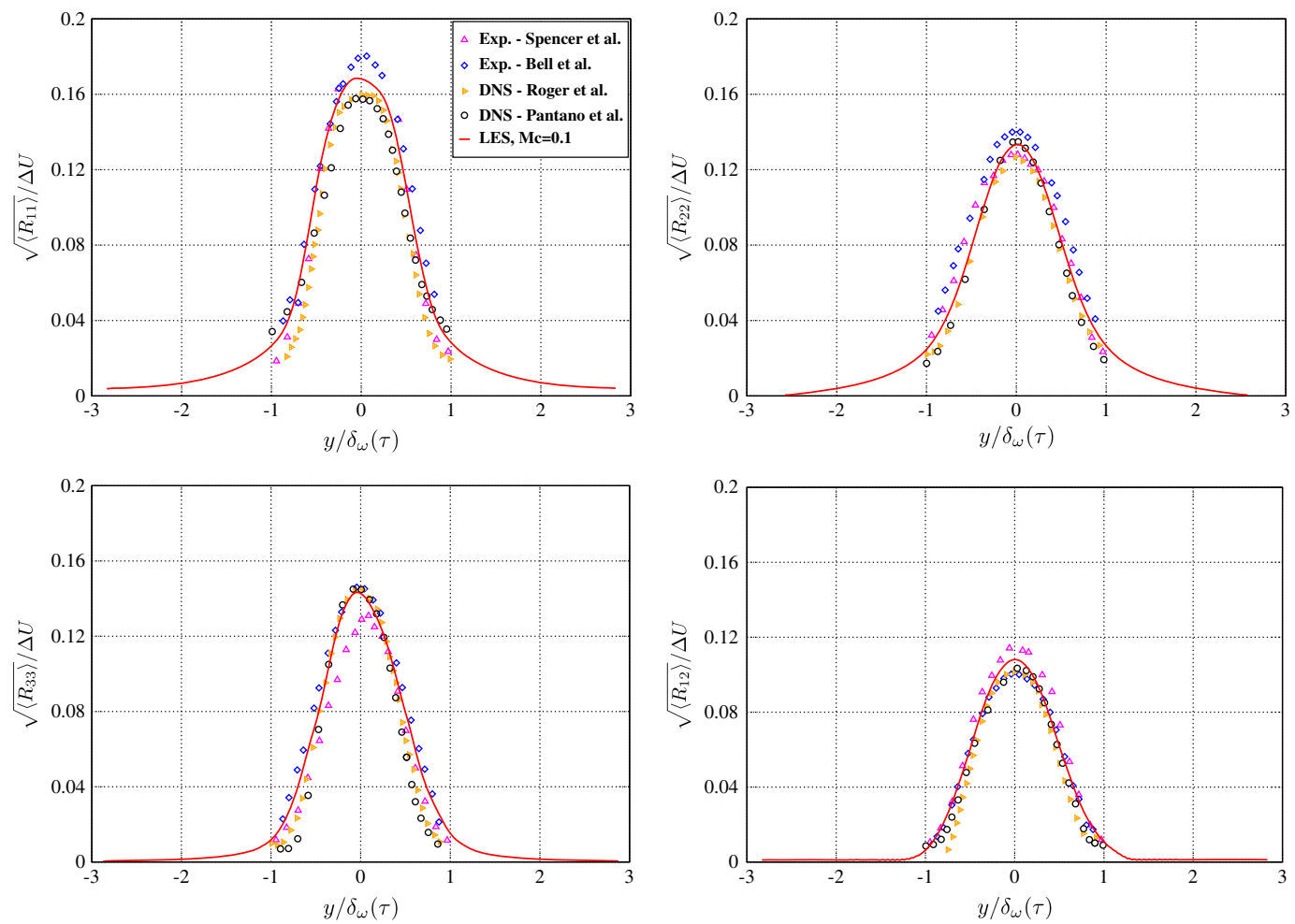

Figure 8. Comparison of the normalized Reynolds stress tensor, $R_{i j}=\left\langle\rho u_{i}^{\prime} u_{j}^{\prime}\right\rangle /\langle\rho\rangle$.

Table II. Comparison of peak turbulent intensities of the incompressible mixing layer.

\begin{tabular}{lccc}
\hline & Bell et al. & Pantano et al. & LES-C1 / LES-C2 \\
\hline$M_{c}$ & 0 & 0.3 & $0.1 / 0.3$ \\
$\sqrt{\left\langle R_{11}\right\rangle} / \Delta U$ & 0.18 & 0.155 & 0.17 \\
$\sqrt{\left\langle R_{22}\right\rangle} / \Delta U$ & 0.14 & 0.134 & 0.134 \\
$\sqrt{\left\langle R_{33}\right\rangle} / \Delta U$ & 0.146 & 0.143 & 0.143 \\
$\sqrt{\left\langle R_{12}\right\rangle} / \Delta U$ & 0.10 & 0.103 & 0.106 \\
$\sqrt{\left\langle R_{22}\right\rangle /\left\langle R_{11}\right\rangle}$ & 0.777 & 0.788 & 0.788 \\
$\sqrt{\left\langle R_{12}\right\rangle /\left\langle R_{11}\right\rangle}$ & 0.555 & 0.606 & 0.623 \\
\hline
\end{tabular}




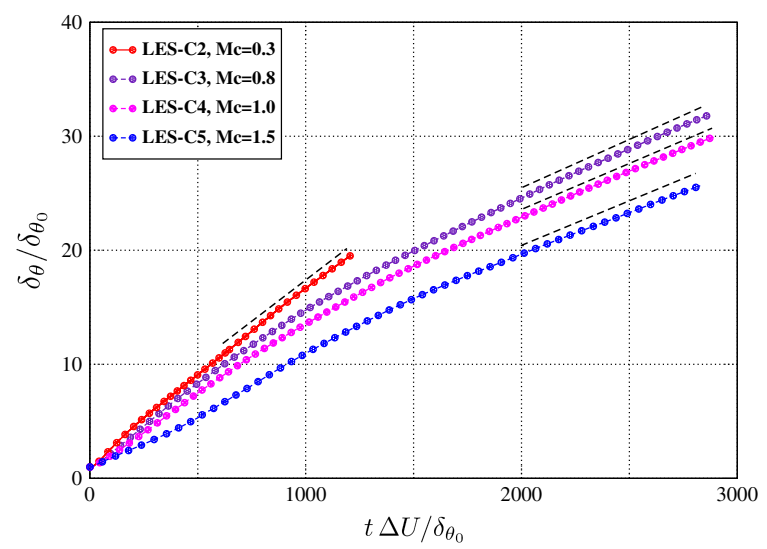

Figure 9. Time evolution of normalized momentum thickness for LES at different convective numbers.

time ( $\tau>2000$ compared to the incompressible one), corresponding to the initial transient, the mixing layer grows quasi-linearly with spread rates of $d\left(\delta_{\theta} / \delta_{\theta_{0}}\right) / d \tau=0.0165,0.0101,0.0084$, and 0.0075 for cases LES-C2, LES-C3, LES-C4, and LES-C5, respectively.

In compressible mixing layers, all assessments of the compressibility effects can be related to the convective Mach number, $M_{c}$, through the compressibility factor, $\Phi=(d \delta / d \tau)_{c} /(d \delta / d \tau)_{i}$, which is the ratio of the compressible growth rate to the incompressible growth rate at the same velocity and temperature ratios. The calculated compressibility factor is significantly less than 1 (the incompressible counterpart), with the ratio of the two being less than 0.43 for $M_{c}>1$. This is consistent with previous findings on the effects of compressibility on mixing-layer growth rate, such as the nonlinear regression fit of Barone [54,55] plotted in Figure 10. This plot shows the ratio of compressible mixing-layer growth to the incompressible mixing-layer growth rate as a function of $M_{c}$, and data from different experiments and previous DNS have been included for comparison. The three higher compressibility cases have growth rates that agree well with previously published data. As already pointed out by Papamoschou [56], the growth rate reduction starts at subsonic values of $M_{c}$ and is evidently completed before $M_{c}$ becomes supersonic (Figure 10). This implies that compressibility takes effect before any shock or expansion waves appear in the flow, in the convective frame of reference.

It is worth noticing that the data in this figure exhibit significant scattering which is partly attributable to the different experimental conditions. As pointed out by Barone et al. [54], one can mention that future investigations should be conducted at higher convective Mach numbers to better determine the asymptotic value of $\Phi$.

Also, the dependence of the turbulent kinetic energy of the shear layer on $M_{c}$ is shown in Figure 11. The simulations show that the turbulence intensity decreases with increasing convective Mach number. The decreased level of energy is responsible for the reduction of the mixing thickness growth rate, as already pointed out by Samimy [57], Vreman et al. [58], and many other LES and DNS studies [42, 44, 59].

\subsection{Flow structures and shocklets}

The invariant of velocity gradient tensor $\mathbf{Q}$ and the corresponding normalized form $\boldsymbol{\Lambda}$ are defined by

$$
\mathbf{Q}=\frac{1}{2}\left[\Omega_{i j} \Omega_{i j}-S_{i j} S_{i j}\right], \quad \boldsymbol{\Lambda}=\frac{\left[\Omega_{i j} \Omega_{i j}-S_{i j} S_{i j}\right]}{\left[\Omega_{i j} \Omega_{i j}+S_{i j} S_{i j}\right]},
$$

where $S_{i j}=\left(u_{i, j}+u_{j, i}\right) / 2, \Omega_{i j}=\left(u_{i, j}-u_{j, i}\right) / 2$.

The iso-surfaces of $\mathbf{Q}$ and $\boldsymbol{\Lambda}$ are plotted for flow visualization of the mixing layers. It is evident that the positive values of $\mathbf{Q}$ and $\boldsymbol{\Lambda}$ represent the vortex-dominated portion of the flow. Three-dimensional perspective views of the iso-surfaces of $\mathbf{Q}$ are presented in Figure 12 for LES-C2 in a self-similar state. The 3-D complex vortex tube structures are clearly evident from these figures. 


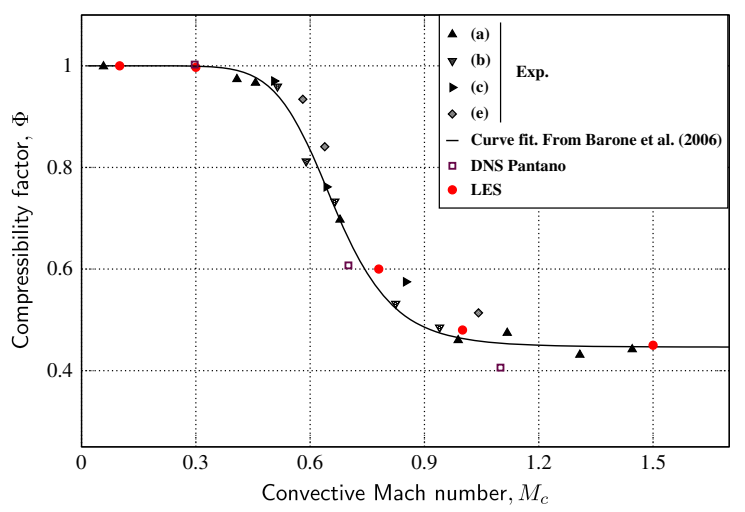

Figure 10. Temporal mixing. Compressibility factor as a function of the convective Mach number from different experimental mixing-layer studies selected by Barone et al. [54]: (a) Bogdanoff [60], and Papamoschou and Roshko [50]; (b) Chinzei et al. [61]; (c) Samimy and Elliott [57, 62]- nonlinear regression curve from [54] with $\Phi\left(M_{c}\right)=1-a_{1}\left[1-1 /\left(1+a_{2} M_{c}^{a_{3}}\right)\right], a_{1}=0.5537, a_{2}=31.79, a_{3}=8.426$; (d) Gruber et al. [63]. LES computations with WENO7fi (red solid circles) for $M_{c}=0.1,0.3,0.8,1.0,1.5$.

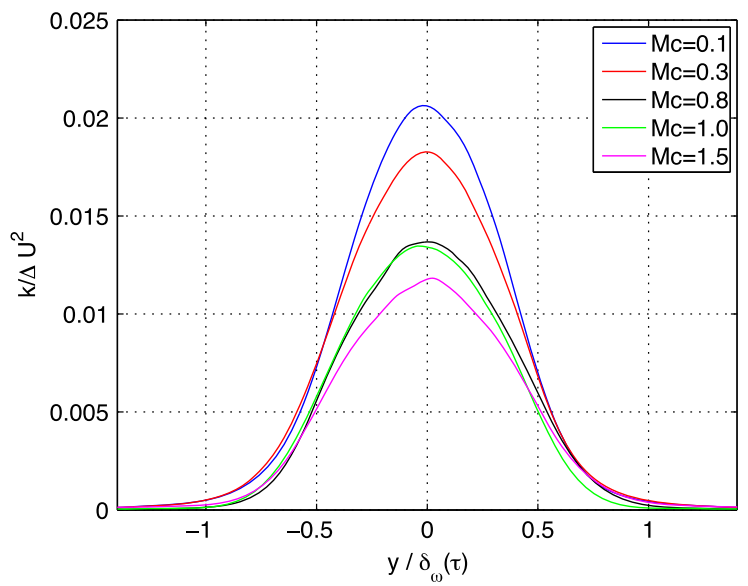

Figure 11. Normalized turbulent kinetic energy at different convective Mach numbers.

With regard to the highly compressible case $M_{c}=1.5$, the complexity of the three-dimensional flow structure leads to difficulties in the identification of shocklets (see Figure 13) . One good method to identify the location of a shock is to use a Schlieren-based technique to portray shocks and, even more, weak discontinuities in the fluid (see [29] for more details about flow visualization). Because, in our case, the initial density is uniform, we used Schlieren on the basis of the dilatation of the velocity field $\nabla \mathbf{u}$ to highlight the eddy shocklets (see Figure 14). Note that shocklets start to appear at Mach number less than unity, that is, in the lower part of the transonic regime. As expected, for higher Mach numbers, the shocklets become stronger and are preferentially organized in oblique waves (see Figure 14), corresponding to stationary inviscid shocks at dominant propagation directions, $\theta$, and a nominal Mach number, $M_{n}=\Delta U /(2 c)$, where $c$ is the speed of sound in the unperturbed region. Avital et al. [64,65] provide a correlation used to find the propagation angle corresponding to the most perturbed waves. It is given by $M_{c} \operatorname{Cos} \theta_{t h}=0.6$. for $M_{c}=1.5, \theta_{t h}=66^{\circ}$. In our case, a visual measurement of the oblique wave angle gives an approximate value of $\theta_{\text {sim }} \simeq 65^{\circ}$, which is very close to the predicted value.

From the present computation, it can be seen that the oblique structures start to occur at convective Mach numbers less than unity. These structures are related to compression waves emanating from the shear layer, and also the existence of other perturbing pressure disturbances leads to an enhanced mixing through the creation of streamwise vortices. 

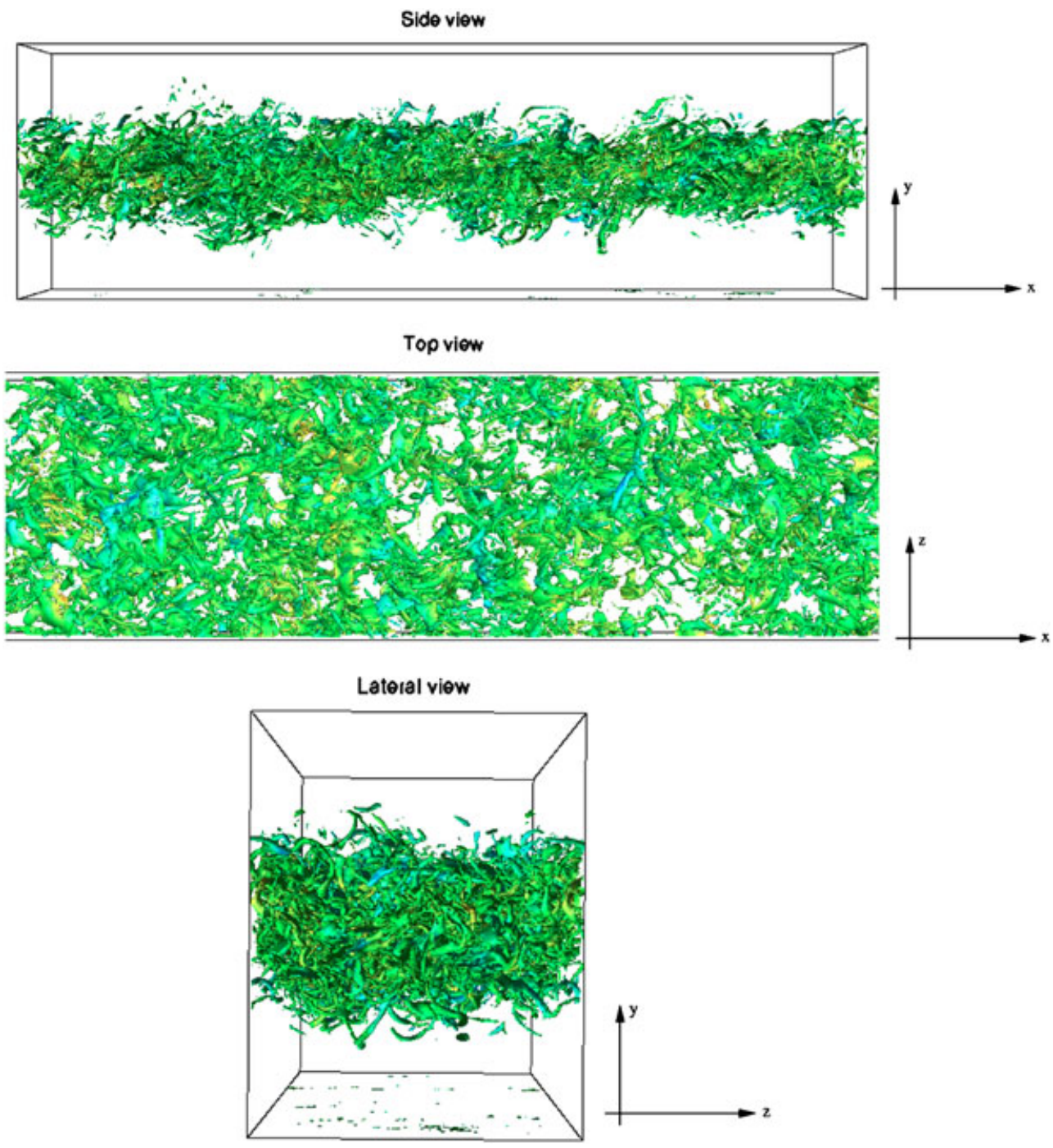

Figure 12. Iso-surfaces of $\mathbf{Q}=0.01, \mathbf{Q}_{\max }$ at $\tau=1000$, LES-C2.

\section{CONCLUDING REMARKS}

This paper illustrates some recent progress in computations of compressible turbulence with the use of a high-order spatial scheme on a LES model for temporally evolving turbulent mixing layers. Results obtained including flow visualization, streamwise velocities, fluctuating velocities, and Reynolds stresses agree well with experimental results. The current LES agree with the previous DNS of the mixing layer by Vreman et al. [58] and Freund et al. [66], which shows decreased turbulence production with increasing $M_{c}$.

The present study serves as a validation and performance of the improved filter schemes of Yee and Sjögreen [7] on a representative complex compressible turbulent flow consisting of a wide range of flow speeds. All the computations use the Ducros et al. splitting of the inviscid flux derivatives and WENO7fi with $\bar{\kappa}$ and $\kappa=0.7$ described in Section 2.2.1. In all $M_{c}$ cases, no tuning of WENO7fi scheme parameters were needed. LES comparison between WENO7fi, WENO5, and WENO7 for the TML is reported in [18]. Studies indicated that WENO7fi compares well with experimental data and published DNS work. For all the considered $M_{c}$ cases, solutions by WENO5 and WENO7 compared poorly with experimental data and DNS computations. The comparative study between WENO7fi, WENO5, and WENO7 is reported in [18] was the first step in determining the suitable order of the filter schemes to be used for the current physics-based study. 

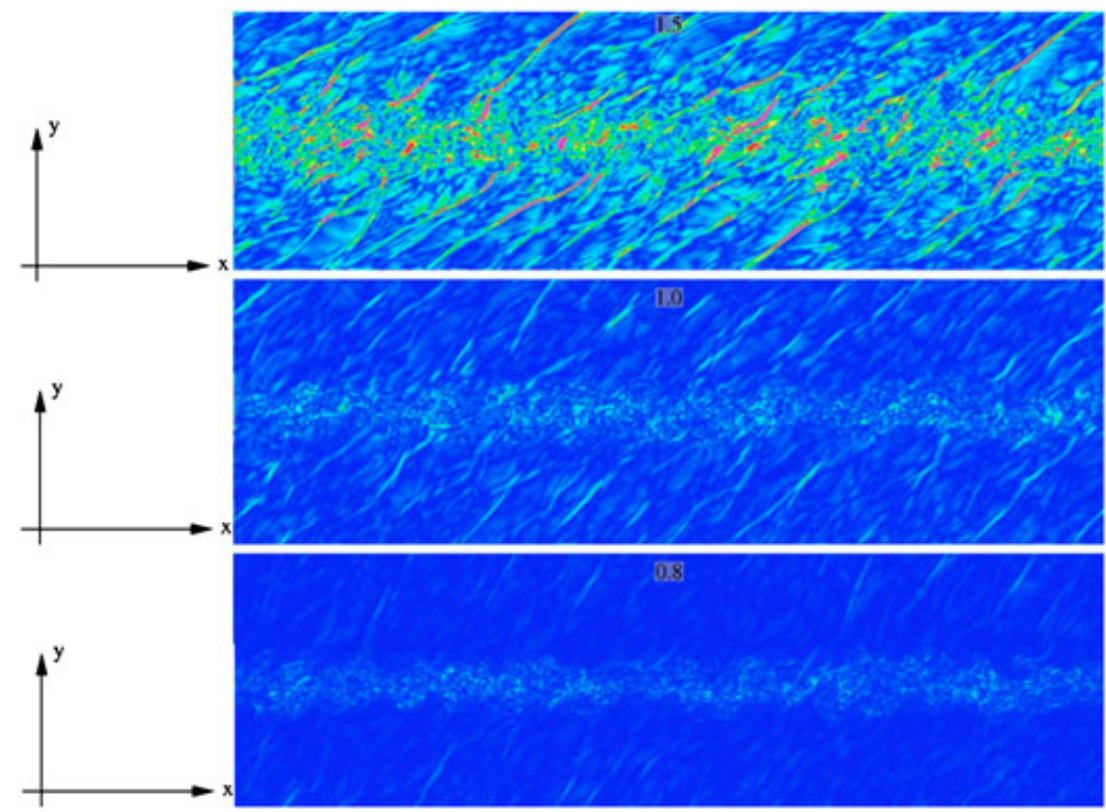

Figure 13. Instantaneous dilatation of the flow field at $\tau=1000$ for three different convective Mach numbers $\left(M_{c}=1.5,1.0\right.$, and 0.8 from top to bottom). Note that the plots are based on the nonlinear dimensionless variable, $\phi=1-\tanh \left[\kappa \nabla \cdot \mathbf{u} /(\nabla \cdot \mathbf{u})_{\max }\right]$, and the parameter $\kappa=0.5$ governs the amplification of small gradients. A value of $\kappa$ close to 15 provides good results.

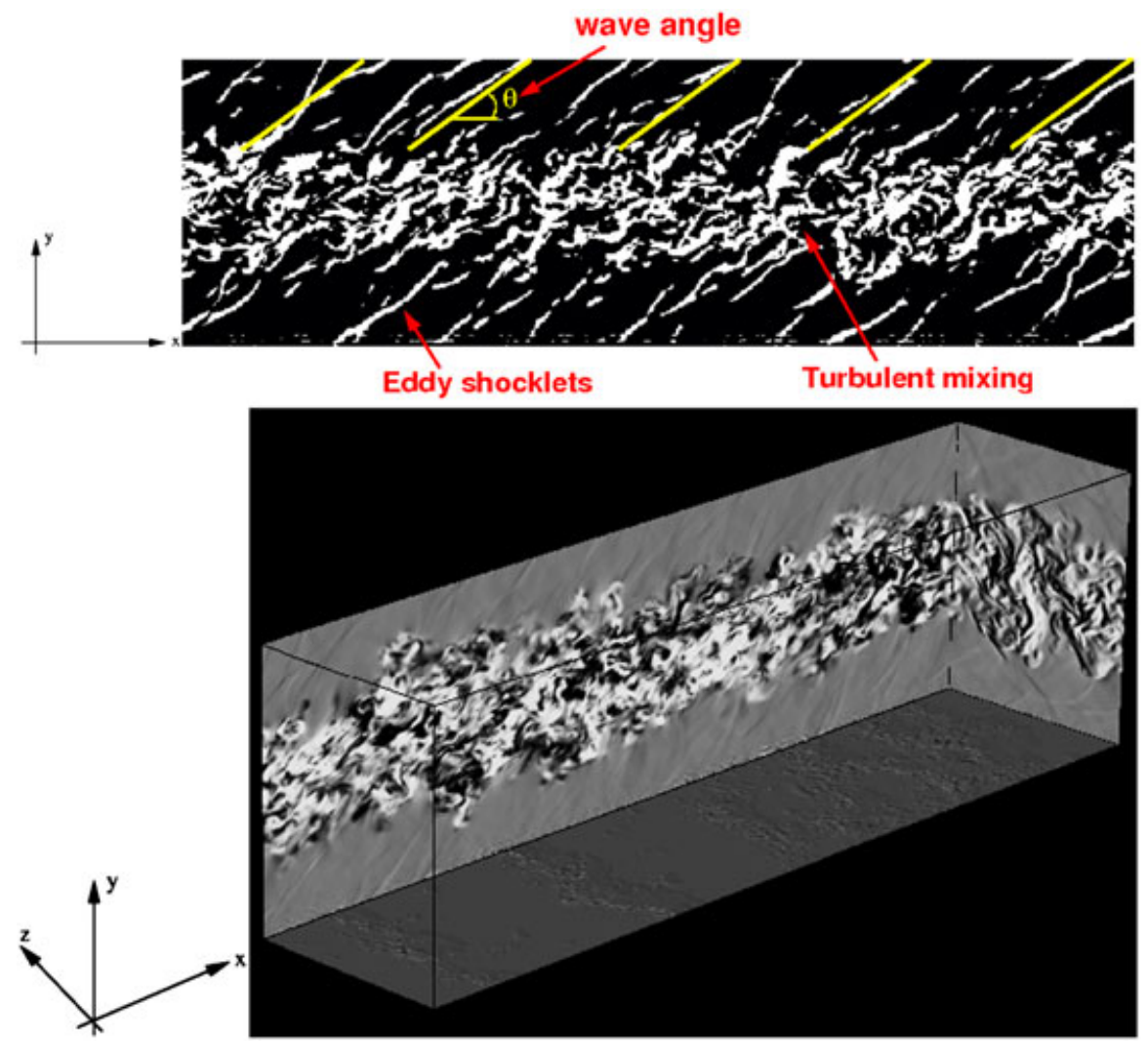

Figure 14. Instantaneous numerical Schlieren pictures at $\tau=2000$, LES-C5. 
The same high-order filter scheme is being used for the simulation of two much higher $M_{c}$ cases of $M_{c}=2,3$. The computational box size, especially in the $y$-direction, has to be doubled or more. A finer grid is also needed to obtain an accurate and stable solution. These computations are many times more CPU-intensive than the lower $M_{c}$ cases. Results will be reported in a forthcoming paper.

\section{ACKNOWLEDGEMENTS}

The authors wish to express their gratitude to T. Sandstrom and C. Henze of the Visualization Group, and A. Lazanoff and J. Chang of the Scientific Consultant Group, Code TN, NASA Ames for their help. Special thanks to M. Rogers and A. Wray for their valuable discussion during the course of this research. The support of the DOE/SciDAC SAP grant DE-AI02-06ER25796 is acknowledged. Part of the work by the second author was performed under the NASA Fundamental Aeronautics Hypersonic Program. Work by the third author was performed under the auspices of the U.S. Department of Energy by Lawrence Livermore National Laboratory under Contract DE-AC52-07NA27344.

\section{APPENDIX A: SUBGRID MODEL}

The most widely used and the simplest model is Smagorinsky's model [31], which employs an eddy viscosity hypothesis to express the subgrid-scale stress as

$$
\tau_{i j}-\frac{1}{3} \tau_{k k} \delta_{i j}=-2 \mu_{t}\left(\widetilde{S}_{i j}-\frac{1}{3} \widetilde{S}_{k k} \delta_{i j}\right) .
$$

The eddy viscosity, $\mu_{t}$, is modeled according to

$$
\mu_{t}=\bar{\rho} C_{s} \Delta^{2}|\widetilde{S}|,
$$

where $C_{s}$ is the Smagorinsky constant and $|\widetilde{S}|$ is defined as

$$
|\widetilde{S}|=\left(2 \widetilde{S}_{i j} \widetilde{S}_{i j}\right)^{1 / 2} \text {. }
$$

The model for the isotropic part of the subgrid-scale stress was proposed by Yoshizawa [67] as

$$
\tau_{k k}=2 C_{I} \bar{\rho} \Delta^{2}|\widetilde{S}|^{2} .
$$

According to Erlebacher et al. [68], $\tau_{k k}$ can be neglected in flows where turbulent Mach number, $M_{t}=\frac{\sqrt{3} u_{r m s}}{\langle a\rangle}$, is less than 0.4 .

The dynamic procedures have been developed to evaluate the parameters used in the subgrid models by utilizing the information provided by the resolved scales. The original procedure was developed by Germano et al. [34] and later modified by Lilly [32] for incompressible flows. Moin et al. [69] generalized the dynamic procedure for compressible flows. The procedure is as follows: The subgrid-scale stress for compressible flows, which is defined in Equation (11), can be rewritten as

$$
\tau_{i j}=\overline{\rho u_{i} u_{j}}-\left(\frac{\overline{\rho u_{i}} \overline{\rho u_{i}}}{\bar{\rho}}\right) .
$$

Now, the field $\widetilde{u}_{i}$ is considered as an instantaneous field, and a test filter with the filter kernel $\widehat{G}(\vec{x}-\vec{y}, 2 \Delta)$ is applied to the LES-filtered Navier-Stokes equation to obtain the resolved turbulent stress,

$$
L_{i j}=\left(\widehat{\bar{\rho} \widetilde{u}_{i} \widetilde{u}_{j}}\right)-\frac{\widehat{\rho} \widehat{\widehat{u}}_{i} \widehat{\rho} \widehat{\hat{u}}_{j}}{\widehat{\bar{\rho}}}
$$

where $\widehat{q}$ denotes the test-filtered variable $q$. The subtest stresses are defined by

$$
T_{i j}=\widehat{\widehat{\rho u_{i} u_{j}}}-\frac{\widehat{\rho u_{i}} \widehat{\rho u_{j}}}{\widehat{\bar{\rho}}}
$$


From Equations (A.5), (A.7), and (A.6), we obtain the Germano identity

$$
L_{i j}=T_{i j}-\widehat{\tau_{i j}}
$$

In the above equation, the two terms on the r.h.s. can be modeled according to Smagorinsky. The term in the l.h.s. can be explicitly calculated by applying the test filter to the simulation results that were obtained using the first filter.

The anisotropic part of the subgrid stress given in Equation (A.7) can be modeled according to Smagorinsky as

$$
T_{i j}-\frac{1}{3} T_{l l} \delta_{i j}=-2 C_{s} \widehat{\bar{\rho}} \widehat{\Delta}^{2}|\widehat{S}| \widehat{S_{i j}^{\star}}
$$

with $\widetilde{S}_{i j}^{\star}=\widetilde{S}_{i j}-\frac{1}{3} \widetilde{S}_{l l} \delta_{i j}$. The isotropic part of the subgrid stress given in Equation A.7 can be modeled according to Yoshizawa as

$$
T_{l l}=2 C_{I} \widehat{\bar{\rho}} \widehat{\Delta}^{2}|\widehat{\widehat{S}}|^{2} .
$$

After applying the test filter to the subgrid stress tensor of $\widetilde{u}_{i}$ (Equation (A.1)) and substituting it along with Equation (A.9) into Equation (A.8), we obtain

$$
\begin{gathered}
\underbrace{L_{i j}-\frac{1}{3} L_{l l} \delta_{i j}}_{L_{i j}^{C_{S}}}=C_{s} \underbrace{\left[-2 \widehat{\bar{\rho}} \widehat{\Delta}^{2}|\widehat{\widetilde{S}}| \widehat{\widehat{S}_{i j}^{\star}}+2 \Delta^{2}\left(\left(\widehat{\bar{\rho}|\widetilde{S}| \widetilde{S}_{i j}}\right)-\frac{1}{3}\left(\widehat{\bar{\rho}|\widetilde{S}| \widetilde{S}_{l l}}\right) \delta_{i j}\right)\right]}_{M_{i j}^{C_{S}}}, \\
L_{l l}=C_{I} \underbrace{\left[2 \widehat{\bar{\rho}} \widehat{\Delta}^{2}|\widehat{\widetilde{S}}|^{2}-2 \Delta^{2}\left(\widehat{\bar{\rho}|\widetilde{S}|^{2}}\right)\right]}_{M_{l l}^{C_{I}}} .
\end{gathered}
$$

The above equations can be rewritten in compact form as

$$
L_{i j}^{C_{s}}=C_{s} M_{i j}^{C_{s}}, \quad L_{l l}=C_{I} M_{l l}^{C_{I}} .
$$

Modifying the original approach by Germano to find $C_{s}$ and $C_{I}$ from the above equations, Lilly [32] introduced the least square method which gives

$$
C_{s}=\frac{\left\langle L_{i j}^{C_{s}} M_{i j}^{C_{s}}\right\rangle_{H}}{\left\langle M_{i j}^{C_{s}} M_{i j}^{C_{S}}\right\rangle_{H}}, \quad C_{I}=\frac{\left\langle L_{l l}\right\rangle_{H}}{\left\langle M_{l l}^{C_{I}}\right\rangle_{H}} .
$$

In the above equations, as we can see, an averaging is done in the homogenous direction. This is to avoid excessively large local values of $C_{s}$ and $C_{I}$ which may destabilize the numerical simulation.

\section{APPENDIX B: ALGORITHM FOR TURBULENCE INITIALIZATION}

(a) Choose length scales in each direction $L_{x}=n_{x} \Delta x, L_{y}=n_{y} \Delta y$, and $L_{z}=n_{z} \Delta z$

(b) Choose a filter width $N_{f_{\alpha}} \geqslant 2 n_{\alpha}, \alpha=x, y, z$

(c) Initialize and store random fields $\mathcal{R}_{\alpha}$ with zero means and unity variances of the dimension $\left[-N_{f_{x}}: N_{f_{x}},-N_{f_{y}}+1: N_{f_{y}}+N_{y},-N_{f_{z}}+1: N_{f_{z}}+N_{z}\right]$, where $N_{y} \times N_{z}$ is the number of the mesh points in the $y z$ plane

(d) Calculate filter coefficients $b(i, j, k)$, where $b_{i, j, k}=b_{i} \cdot b_{j} \cdot b_{k}$,

$$
b_{k} \approx \frac{\tilde{b}_{k}}{\left(\sum_{j=-N f}^{N f} \tilde{b}_{j}^{2}\right)^{1 / 2}}, \quad \text { and } \quad \tilde{b}_{k}=\exp \left(-\frac{\pi k^{2}}{2 n^{2}}\right)
$$


(e) Apply the filter operation for $j=1, \ldots \ldots, N_{y}, k=1, \ldots, N_{z}$

$$
\mathcal{U}_{\alpha}(j, k)=\sum_{i^{\prime}=-N_{f_{x}}}^{N_{f_{x}}} \sum_{j^{\prime}=-N_{f_{y}}}^{N_{f_{y}}} \sum_{k^{\prime}=-N_{f_{z}}}^{N_{f_{z}}} b\left(i^{\prime}, j^{\prime}, k^{\prime}\right) \mathcal{R}_{\alpha}\left(i^{\prime}, j+j^{\prime}, k+k^{\prime}\right)
$$

which results in the two-dimensional arrays of spatially correlated data $\mathcal{U}_{\alpha}$

(f) Perform the following coordinate transformation to obtain $\mathcal{U}_{\alpha}^{\prime}(j, k)=a_{i j} \mathcal{U}_{\alpha}(j, k)$, with the prescribed Reynolds stress tensor

$$
a_{i j}=\left(\begin{array}{ccc}
\left(R_{11}\right)^{1 / 2} & 0 & 0 \\
R_{21} / a_{11} & \left(R_{22}-a_{21}^{2}\right)^{1 / 2} & 0 \\
R_{31} / a_{11} & \left(R_{32}-a_{21} a_{31}\right) / a_{22} & \left(R_{33}-a_{31}^{2}-a_{32}^{2}\right)^{1 / 2}
\end{array}\right)
$$

(g) Calculate $u_{\alpha}(j, k)=\bar{u}_{\alpha}(j, k)+\mathcal{U}^{\prime}{ }_{\alpha}(j, k)$ for the first $(j, k)$ plane

(h) Discard the first $y, z$ plane of $\mathcal{R}_{\alpha}$ and shift the whole data $\mathcal{R}_{\alpha}(i, j, k):=\mathcal{R}_{\alpha}(i+1, j, k)$

(i) Generate new random numbers to fill the plane $\mathcal{R}_{\alpha}\left(N_{f_{x}}, j, k\right)$

(j) Repeat the steps $(e)$ to $(i)$ for each mesh point in the $x$-direction

\section{REFERENCES}

1. Yee HC, Torczynski JR, Morton SA, Visbal MR, Sweby PK. On spurious behavior of CFD simulations. In AIAA 97-1869, Proceedings of the 13th AIAA Computational Fluid Dynamics Conference June 29 - July 2, 1997, Vol. 30, 1999; 675-711. Snowmass, CO.; also International Numerical Methods in Fluids.

2. Yee HC, Sweby PK. Dynamics of numerics \& spurious behaviors in CFD computations. RIACS Technical Report 97.06, Beijing, China, June 1997. Keynote paper, 7th ISCFD Conference, Sept. 15-19, 1997.

3. Yee HC, Sjögreen B. Designing adaptive low dissipative high order schemes for long-time integrations. In Turbulent Flow Computation, Drikakis D, Geurts B (eds). Kluwer Academic Publisher: Dordrecht, U.K, 2002. also RIACS Technical Report TR01-28, Dec. 2001, NASA American Research Center.

4. Yee HC. Building blocks for reliable complex nonlinear numerical simulations. In Turbulent Flow Computation, Drikakis D, Geurts B (eds). Kluwer Academic Publisher: Dordrecht, U.K, 2002. also RIACS Technical Report TR01-28, Dec. 2001, NASA American Research Center.

5. Yee H, Sjögreen B. Development of low dissipative high order filter schemes for multiscale Navier-Stokes/MHD systems. Journal of Computational Physics 2007; 225:910-934.

6. Yee HC, Sjögreen B, Barone M. High order numerical schemes for hypersonic flow simulations. VKI Lecture Series. Course on Hypersonic Entry and Cruise Vehicles, 30 June - 3 July 2008.

7. Yee HC, Sjögreen B. High order filter methods for wide range of compressible flow speeds. Proceedings of ICOSAHOM 09 (International Conference on Spectral and High Order Methods), Trondheim, Norway. June 22-26, 2009.

8. Sjögreen B, Yee HC. Variable high order multiblock overlapping grid methods for mixed steady and unsteady multiscale viscous flows. Communication in Computational Physics 2009; 5:730-744.

9. Wang W, Shu CW, Yee HC, Sjögreen B. High order finite difference methods with subcell resolution for advection equations with stiff source terms. Journal of Computational Physics 2012; 231:190-214.

10. Yee H, Sjögreen B, Shu CW, Wang W, Magin T, Hadjadj A. On numerical methods for hypersonic turbulent flows. PROCEEDINGS of ESA 7th Aerothermodynamics Symposium, Site Oud Sint-Jan, Brugge, Belgium, May 2011.

11. Yee HC, Sjögreen B. Local flow sensors in controlling numerical dissipations for a wide spectrum of flow speed and shock strength. in preparation.

12. Sjögreen B, Yee HC. Multiresolution wavelet based adaptive numerical dissipation control for shock-turbulence computation. Journal of Scientific Computing 2004; 20:211-255.

13. Olsson P, Oliger J. Energy and maximum norm estimates for nonlinear conservation laws. RIACS Technical Report 94.01, 1994, NASA American Research Center.

14. Yee HC, Vinokur M, Djomehri MJ. Entropy splitting and numerical dissipation. Journal of Computational Physics 2000; 162:33-81.

15. Ducros F, Laporte F, Souleres T, Guinot V, Moina P, Caruelle B. High-order fluxes for conservative skew-symmetriclike schemes in structured meshes: Application to compressible flows. Journal of Computational Physics 2000; 16:114-139.

16. Sjögreen B, Yee HC. On skew-symmetric splitting of the Euler equations. Proceedings of the EUNUMATH-09 Conference. June 29 - July 2, 2009, to appear.

17. Wang W, Yee HC, Sjögreen B, Magin T, Shu CW. Construction of low dissipative high-order wellbalanced filter schemes for nonequilibrium flows. Journal of Computational Physics to appear 2010. DOI: 10.1016/j.jcp.2010.04.033, 2010. 
18. Yee H, Sjögreen B, Hadjadj A. Comparative Study of high order schemes for LES of temporal-evolving mixing layers. Proceedings of ASTRONUM-2010, San Diego, Calif. June 13-18, 2010.

19. Sagaut P, Germano M. On the filtering paradigm for LES of flows with discontinuities. Journal of Turbulence 2005; 6:1-9.

20. Yee HC, Sandham ND, Djomehri MJ. Low dissipative high order shock-capturing methods using characteristic-based filters. Journal of Computational Physics 1999; 150:199-238.

21. Yee H, Sjögreen B. Efficient low dissipative high order scheme for multiscale MHD flows, II: minimization of $\operatorname{div}(\mathrm{B})$ numerical error. RIACS Technical Report TR03.10, NASA Ames Research Center, 2006. July, 2003, ; also, Journal of Scientific Computing, 29 115-164.

22. Kotov D, Yee HC, Sjögreen B, Wang W, Shu CW. Performance of four high order shock-capturing schemes for stiff source terms with discontinuities: preliminary results, October 2011. CTR Annual Technical Brief, Stanford University.

23. Yee HC, Kotov D, Sjögreen B, Wang W, Shu CW. Numerical dissipation and wrong propagation speed of discontinuities for stiff source terms. Proceedings of the ASTRONUM-2011, Valencia, Spain, June 2011.

24. Jiang G-S, Shu C-W. Efficient implementation of weighted ENO schemes. Journal of Computational Physics 1996; 126:202-228.

25. Roe PL. Approximate Riemann solvers, parameter vectors, and difference schemes. Journal of Computational Physics 1981; 43:357-372.

26. Lo S-C, Blaisdell GA, Lyrintzis AS. High-order shock capturing schemes for turbulence calculations. International Journal in Numerical Methods in Fluids 2010; 62:473-498.

27. Ducros F, Ferrand V, Nicoud F, Weber C, Darracq D, Gacherieu C, Poinsot T. Large-Eddy simulation of the shock/ turbulence interaction. Journal of Computational Physics 1999; 152:517-549.

28. Li X-S, Gu C-W. An All-speed Roe-type scheme and its asymptotic analysis of low Mach number behaviour. Journal of Computational Physics 2008; 227:5144-5159.

29. Hadjadj A, Kudryavtsev A. Computation and flow visualization in high speed aerodynamics. Journal of Turbulence $2005 ; \mathbf{6}: 33-81$.

30. Farge M, Pellegrino G, Schneider K. Coherent vortex extraction in 3D turbulent flows using orthogonal wavelets. Physics of Review Letters 2001; 5:45011-45014.

31. Smagorinsky J. General circulation experiments with the primitive equations. I. the basic experiment. Monthly Weather Review 1963; 91:99.

32. Lilly DK. A proposed modification of the Germano subgrid-scale closure method. Physics of Fluid 1992; 4:633-635.

33. Deardorff JW. Three dimensional numerical study of the height and mean structure of a heated planetary boundary layer. Boundary Layer Meteorol 1974; 7:81-106.

34. Germano M, Piomelli U, Moin P, Cabot WH. A dynamic subgrid-scale eddy viscosity model. Physics of Fluid 1991; A 3:1760-1765.

35. Sagaut P. Large eddy simulation of turbulent flows. Springer: Berlin, 2001.

36. Pope SB. Turbulent flows. Cambridge University Press: Cambridge, 2000.

37. Pope SB. Ten questions concerning the large eddy simulation of turbulent flows. New Journal of Physics 2004; 6:35-40.

38. Rogallo RS, Moin P. Numerical simulation of turbulent flows. Ann. Rev. Fluid Mech. 1984; 18:99-137.

39. Galperin B, Orszag SA. Large eddy simulation of complex engineering and geophysical flows. Cambridge University Press: Cambridge, U.K, 1993.

40. Lesieur M, Metais O. New trends in large-eddy simulations of turbulence. Annual Review of Fluid Mechanics 1996; 28:45-82.

41. Meneveau C, Katz J. Scale-invariance and turbulence models for Large-Eddy simulation. Annual Review of Fluid Mechanics 2000; 32:1-32.

42. Foysi H, Sarkar S. The compressible mixing layer: an LES study. Theoretical and Computational Fluid Dynamics 2010. DOI: $10.1007 / \mathrm{s} 00162-009-0176-8$.

43. Mahle I, Sesterhenn J, Friedrich R. Turbulent mixing in temporal compressible shear layers involving detailed diffusion processes. Journal of Turbulence 2007; 8:1-2.

44. Pantano C, Sarkar S. A study of compressible effects in the high-speed turbulent shear layer using direct simulation. Journal of Fluid Mechanics 2002; 451:329-371.

45. Klein M, Sadiki A, Janicka J. A digital filter based generation of inflow data for spatially developing direct numerical or large eddy simulation. AIAA Journal 2003; 186:652-665.

46. Bell JH, Mehta RD. Development of a two-stream mixing layer from tripped and untripped boundary layers. AIAA J. 1990; 28(12):2034-2042.

47. O'Neill PL, Nicolaides D, Honnery D, Soria J. Autocorrelation functions and the determination of integral length with reference to experimental and numerical data. Proc. of the 15th Australasian Fluid Mech. Conf., The Univ. of Sydney, Australia, Dec. 13, 2004.

48. Rogers MM, Moser RD. Direct simulation of a self-similar turbulent mixing layer. Phys. Fluids 1994; 6:903-923.

49. Spencer BW, Jones B. Statistical investigation of pressure and velocity fields in the turbulent two-stream mixing layer. AIAA Paper 1971:71-613.

50. Papamoschou D, Roshko A. The compressible turbulent shear layer: an experimental study. Journal of Fluid Mechanics 1988; 197:453-477. 
51. Elliott GS, Samimy M. Compressibility effects in free shear layers. Physics of Fluid 1990; 2:1231-1240.

52. Barre S, Braud P, Chambres O, Bonnet JP. Influence of inlet pressure conditions on supersonic turbulent mixing layers. Experimental Thermal and Fluid Science 1997; 14:68-74.

53. Chambres O, Barre S, Bonnet JP. Detailed turbulence characteristics of a highly compressible supersonic turbulent plane mixing layer. Journal of Fluid Mechanics 1998. Submitted.

54. Barone MF, Oberkampf WL, Blottner FG. Validation case study: prediction of compressible turbulent mixing layer growth rate. AIAA Journal 2006; 44:1488-1497.

55. Oberkampf WL, Barone MF. Measures of agreement between computation and experiment: validation metrics. Journal of Computational Physics 2006; 217:5-36.

56. Papamoschou D. Model for entropy production and pressure variation in confined turbulent mixing layer. AIAA Journal 1993; 31:1643-1650.

57. Samimy M, Reeder MF, Elliott GS. Compressibility effects on large structures in free shear flows. Physics of Fluid 1992; 4:1251-1258.

58. Vreman B, Sandham N, Luo KH. Compressible mixing layer growth rate and turbulence characteristics. Journal of Fluid Mechanics 1996; 320:235-258.

59. Sandham ND, Reynolds WC. A numerical investigation of the compressible mixing layer, 1989. Stanford Rep. TF-45.

60. Bogdanoff DW. Compressibility effects in turbulent shear layers. AIAA Journal 1983; 21:926-927.

61. Chinzei N, Masua G, Komuro T, Murakami A, Kudou K. Spreading of two-stream supersonic turbulent mixing layers. Physics of Fluid 1986; 2:1345-1347.

62. Samimy M, Elliot GS. Effect of compressibility on the characteristics of free shear layer. AIAA Journal 1990; 28:439-445.

63. Gruber MR, Messersmith NL, Dutton JC. Three dimensional velocity field in a compressible mixing layer. AIAA Journal 1993; 31:2061-2067.

64. de Avital EJ, Sandham ND, Luo KH. Mach wave radiation by mixing layers. Part I: analysis of the sound field. Theoretical and Computational Fluid Dynamics 1998; 12:73.

65. de Avital EJ, Sandham ND, Luo KH. Mach wave radiation by mixing layers. Part II: analysis of the sound field. Theoretical and Computational Fluid Dynamics 1998; 12(91):91-108.

66. Freund JB, Lele SK, Moin P. Compressibility effects in a turbulent annular mixing layer. Part 1. Turbulence and growth rate. Journal of Fluid Mechanics 2000; 421:229-267.

67. Yoshizawa A. Statistical theory for compressible turbulent shear flows, with the application to subgrid modeling. Physics of Fluid 1986; 29:2152.

68. Erlebacher G, Hussaini MY, Speziale CG, Zang TA. Toward the large eddy simulation of compressible turbulent flows. J. Fluid Mech. 1992; 238:155-185.

69. Moin P, Squires K, Cabot W, Lee S. A dynamic subgrid scale model for compressible turbulence and scalar transport. Physics of Fluid 1991; 3:2746-2757. 